\title{
A DISCRETE-TIME APPROXIMATION FOR DOUBLY REFLECTED BSDES
}

\author{
JEAN-FRANÇOIS CHASSAGNEUX, ${ }^{*}$ Université Paris Diderot - Paris 7, PMA, \\ and ENSAE-CREST
}

\begin{abstract}
We study the discrete-time approximation of doubly reflected backward stochastic differential equations (BSDEs) in a multidimensional setting. As in Ma and Zhang (2005) or Bouchard and Chassagneux (2008), we introduce the discretely reflected counterpart of these equations. We then provide representation formulae which allow us to obtain new regularity results. We also propose an Euler scheme type approximation and give new convergence results for both discretely and continuously reflected BSDEs.
\end{abstract}

Keywords: Reflected BSDEs; discrete-time approximation schemes; game option; regularity

2000 Mathematics Subject Classification: Primary 65C99; 60H35; 60G40

\section{Introduction}

The main motivation of this paper is the discrete-time approximation of backward stochastic differential equations (BSDEs) with two reflecting barriers, also known as doubly reflected BSDEs:

$$
\begin{gathered}
Y_{t}=g\left(X_{T}\right)+\int_{t}^{T} f\left(X_{u}, Y_{u}, Z_{u}\right) \mathrm{d} u-\int_{t}^{T}\left(Z_{u}\right)^{\top} \mathrm{d} W_{u}+\int_{t}^{T} \mathrm{~d} K_{u}^{+}-\int_{t}^{T} \mathrm{~d} K_{u}^{-}, \\
l\left(X_{t}\right) \leq Y_{t} \leq h\left(X_{t}\right) \quad \text { for all } t \in[0, T], \text { almost surely (a.s.), } \\
\int_{0}^{T}\left(Y_{s}-l\left(X_{s}\right)\right) \mathrm{d} K_{s}^{+}=\int_{0}^{T}\left(Y_{s}-h\left(X_{s}\right)\right) \mathrm{d} K_{s}^{-}=0,
\end{gathered}
$$

where $f$ and $g$ are Lipschitz continuous functions, $h$ and $l$ are smooth functions (say $C_{b}^{2}$ ), and the process $X$ is the solution of a forward SDE

$$
X_{t}=X_{0}+\int_{0}^{t} b\left(X_{s}\right) \mathrm{d} s+\int_{0}^{t} \sigma\left(X_{s}\right) \mathrm{d} W_{s},
$$

with $b$ and $\sigma$ Lipschitz continuous.

These equations can be considered as extensions of simply reflected BSDEs, which are related to the optimal stopping problem (American option in finance) (see, e.g. [9]), and whose numerical approximation has been widely studied (see, e.g. [2], [3], [5], and [15]).

Existence and uniqueness of solutions to (1.1a)-(1.1c) were first studied by Cvitanić and Karatzas [7]. There have been a lot of contributions on this subject since then, consisting essentially in weakening the assumptions for the existence of a solution of (1.1a)-(1.1c); see,

Received 8 June 2007; revision received 29 January 2009.

* Postal address: ENSAE, Timbre J120, 3 avenue Pierre Larousse, 92245 Malakoff Cedex, France.

Email address: chassagneux@ensae.fr

The author would like to thank Bruno Bouchard for fruitful discussions. 
e.g. [1] and the references therein. In economics, Cvitanić and Karatzas [7], among others, showed that these equations are related to stochastic stopping games (Dynkin games) and Ma and Cvitanić [14] connected them to the pricing of game options (or Israeli options), introduced in [12].

In this Markovian setting, Cvitanić and Ma [14] showed that the solution of (1.1a)-(1.1c) is associated to variational inequalities (or the obstacles problem) of the type

$$
\begin{gathered}
(u-l) \wedge\left\{(u-h) \vee-\left[\partial_{t} u+b \partial_{x} u+\frac{1}{2} \operatorname{tr}\left(\sigma \sigma^{\top} \partial_{x x} u\right)+f\left(t, x, u, \sigma \partial_{x} u\right)\right]\right\}=0, \\
u(T, x)=g(x),
\end{gathered}
$$

in the sense that $\left(Y_{t}, Z_{t}\right)=\left(u\left(t, X_{t}\right), \partial_{x} u \sigma\left(t, X_{t}\right)\right)$ for $t \in[0, T]$. Thus, studying the discrete-time approximation of (1.1a)-(1.1c) offers alternative numerical methods to estimate the solution of (1.2).

While studying the discrete-time approximation of (1.1a)-(1.1c), it became evident that the techniques we used can be applied to a multidimensional setting. Namely, $Y$ takes values in $\mathbb{R}^{d}$ and each component $Y^{\ell}$ verifies

$$
\begin{gathered}
Y_{t}^{\ell}=g^{\ell}\left(X_{T}\right)+\int_{t}^{T} f^{\ell}\left(X_{u}, Y_{u}, Z_{u}\right) \mathrm{d} u-\int_{t}^{T}\left(Z_{u}^{\ell}\right)^{\top} \mathrm{d} W_{u}+\int_{t}^{T} \mathrm{~d} K_{u}^{\ell+}-\int_{t}^{T} \mathrm{~d} K_{u}^{\ell-}, \\
\int_{0}^{T}\left(Y_{s}^{\ell}-l^{\ell}\left(X_{s}\right)\right) \mathrm{d} K_{s}^{\ell-}=\int_{0}^{T}\left(Y_{s}^{\ell}-h^{\ell}\left(X_{s}\right)\right) \mathrm{d} K_{s}^{\ell-}=0, \quad \ell \in\{1, \ldots, d\},
\end{gathered}
$$

and, a.s., for all $t \leq T, Y_{t}$ is constrained to take values in the domain $\mathcal{O}_{X_{t}}$ where

$$
\mathcal{O}_{x}:=\left\{y \in \mathbb{R}^{d} \mid \text { for all } \ell \in\{1, \ldots, d\}, l^{\ell}(x) \leq y^{\ell} \leq h^{\ell}(x)\right\} .
$$

When $h$ and $l$ are constant, the domain $\mathcal{O}$ is fixed. The results presented here appear then to be a subcase of the results given in [6, Chapter 3], in which the case of a general fixed convex domain was considered; see also Remark 2.4, below.

Following [3] and [15], we first introduce 'discretely reflected' versions of (1.1a)-(1.1c), meaning that condition (1.1b) is imposed only on a deterministic set of times $\Re=\left\{0=: r_{0}<\right.$ $\left.\cdots<r_{\kappa}:=T\right\}$ :

$$
Y_{T}^{\Re}=\tilde{Y}_{T}^{\Re}:=g\left(X_{T}\right) \in \mathcal{O}_{X_{T}},
$$

and, for $j \leq \kappa-1$ and $t \in\left[r_{j}, r_{j+1}\right)$,

$$
\begin{gathered}
\tilde{Y}_{t}^{\Re}=Y_{r_{j+1}}^{\Re}+\int_{t}^{r_{j+1}} f\left(X_{s}, \tilde{Y}_{s}^{\Re}, Z_{s}^{\Re}\right) \mathrm{d} s-\int_{t}^{r_{j+1}}\left(Z_{s}^{\Re}\right)^{\top} \mathrm{d} W_{s}, \\
Y_{t}^{\Re}=\tilde{Y}_{t}^{\Re} \mathbf{1}_{\{t \notin \Re\}}+\mathcal{P}\left(X_{t}, \tilde{Y}_{t}^{\Re}\right) \mathbf{1}_{\{t \in \mathfrak{R}\}},
\end{gathered}
$$

where $\mathcal{P}(x, y)$ is the projection of $y \in \mathbb{R}^{d}$ onto $\mathcal{O}_{x}$.

In the framework of doubly reflected BSDEs, i.e. $d=1$, this corresponds to stochastic stopping games, where the stopping is allowed only on $\Re \backslash\{T\}$.

We now focus on the discrete-time approximation of such equations. As in [3], [5], and [15], we introduce a partition $\pi=\left\{0=: t_{0}<\cdots<t_{n}:=T\right\}$ such that $\Re \subset \pi$, and define $\left(Y^{\pi}, \bar{Z}^{\pi}\right)$ by the backward induction

$$
\begin{aligned}
\bar{Z}_{t_{i}}^{\pi} & =\left(t_{i+1}-t_{i}\right)^{-1} \mathrm{E}\left[\left(W_{t_{i+1}}-W_{t_{i}}\right)\left(Y_{t_{i+1}}^{\pi}\right)^{\top} \mid \mathcal{F}_{t_{i}}\right], \\
\tilde{Y}_{t_{i}}^{\pi} & =\mathrm{E}\left[Y_{t_{i+1}}^{\pi} \mid \mathcal{F}_{t_{i}}\right]+\left(t_{i+1}-t_{i}\right) f\left(X_{t_{i}}^{\pi}, \tilde{Y}_{t_{i}}^{\pi}, \bar{Z}_{t_{i}}^{\pi}\right), \\
Y_{t_{i}}^{\pi} & =\tilde{Y}_{t_{i}}^{\pi} \mathbf{1}_{\left\{t_{i} \notin \Re\right\}}+\mathcal{P}\left(X_{t_{i}}^{\pi}, \tilde{Y}_{t_{i}}^{\pi}\right) \mathbf{1}_{\left\{t_{i} \in \mathfrak{R}\right\}}, \quad i \leq n-1,
\end{aligned}
$$


with terminal condition (recall that $t_{n}=T$ )

$$
\tilde{Y}_{T}^{\pi}=Y_{T}^{\pi}:=g\left(X_{T}^{\pi}\right)
$$

Here, $X^{\pi}$ is the Euler scheme associated to $X$.

As in [3], [5], and [15], we show that the error induced by this scheme,

$$
\max _{i<n} \sup _{t \in\left[t_{i}, t_{i+1}\right)} \mathrm{E}\left[\left|\tilde{Y}_{t}^{\Re}-\tilde{Y}_{t_{i}}^{\pi}\right|^{2}\right]+\mathrm{E}\left[\sum_{i=0}^{n-1} \int_{t_{i}}^{t_{i+1}}\left|Z_{t}^{\Re}-\bar{Z}_{t_{i}}^{\pi}\right|^{2} \mathrm{~d} t\right],
$$

is intimately related to the regularity of the process $\left(Y^{\Re}, Z^{\Re}\right)$, or, equivalently, $\left(\tilde{Y}^{\Re}, Z^{\Re}\right)$, through the quantities

$$
\max _{i<n} \sup _{t \in\left[t_{i}, t_{i+1}\right)} \mathrm{E}\left[\left|\tilde{Y}_{t}^{\Re}-\tilde{Y}_{t_{i}}^{\Re}\right|^{2}\right] \text { and } \mathrm{E}\left[\sum_{i=0}^{n-1} \int_{t_{i}}^{t_{i+1}}\left|Z_{t}^{\Re}-Z_{t_{i}}^{\Re}\right|^{2} \mathrm{~d} t\right] \text {, }
$$

for which we provide new controls in terms of $|\pi|$, the modulus of $\pi$. This is based on a generalization of the representation of $Z^{\Re}$ derived in [3].

In this paper we essentially rely on the basic concepts developed in [3], but we face two new difficulties.

(i) Contrary to [3], where $\mathcal{O}_{x}$ is of the form $\{y \in \mathbb{R}: y \geq \psi(x)\}$, we do not have an exact expression of the projected process $\mathcal{P}\left(X_{t}, \tilde{Y}_{t}^{\Re}\right)$ and the reflection terms are much more intricate to handle.

(ii) In the one-dimensional case, a simple Girsanov transformation allows us to get rid of the Malliavin derivatives of $Y^{\Re}$ and $Z^{\Re}$ which enter the representation formula of $Z^{\Re}$ (see Section 3). This is no longer possible, in general, in our multidimensional setting.

Yet, in the discretely reflected case, we are able to extend the regularity result of [3]. This allows us to show that scheme (1.4a)-(1.4c) has a convergence rate of at least $|\pi|^{1 / 4}$. Under stronger regularity conditions on the boundaries and the coefficients of the SDE solved by $X$, we obtain a convergence rate of at least $|\pi|^{1 / 2}$ (see Subsection 5.3).

Using an approximation argument, we then extend these results to continuously reflected BSDEs. The convergence is obtained under minimal Lipschitz continuity assumptions with a control of order $|\pi|^{1 / 12}$. Under stronger regularity conditions, we extend the one-dimensional result of [15], but without their uniform ellipticity assumption. Namely, we provide an upper bound of order $|\pi|^{1 / 4}$ for the approximation error. When the system of BSDEs is decoupled, which is the most important case for financial applications, we improve it to $|\pi|^{1 / 3}$.

We would like to conclude this introduction by observing that scheme (1.4a)-(1.4c) is obviously not directly implementable since it requires the computation of conditional expectations. The global numerical error is then the sum of the discrete-time approximation error (1.5) and the numerical error induced by the approximation of the conditional expectations. However, this approximation problem is well understood and the authors of [2], [5], and [11], among others, proposed efficient numerical methods, which can be easily adapted to our framework. This paper being already long, we will not detail this part here and focus only on the discretization error. 
The rest of the paper is organized as follows. In Section 2 we define BSDEs which are discretely reflected in a convex domain $\mathcal{O}_{x}$ of the above form. In Section 3 we provide different representations of $Z^{\mathfrak{R}}$ and use them to study the regularity of $\left(Y^{\mathfrak{R}}, \tilde{Y}^{\mathfrak{R}}, Z^{\mathfrak{R}}\right)$ in Section 4. In Section 5 we propose an Euler scheme type approximation of discretely reflected BSDEs and give our main convergence results. Finally, in Section 6 we provide extensions to the continuously reflected case. Appendix A contains the proofs of a priori estimates which are used several times in the paper.

\subsection{Notation}

Let $\mathbb{M}^{n, m}$ be the set of matrices with dimension $n \times m$, we simply write $\mathbb{M}^{d}$ if $m=n=d$. For $z \in \mathbb{M}^{n, m}, z^{i j}$ denotes the $(i, j)$ th component of $z, z^{i}$. denotes the $i$ th row of $z, z^{j}$ denotes the $j$ th column of $z$, and $z^{\top}$ denotes its transposed matrix. The space $L^{p}$ for $p \geq 1$ is the set of random variables $X$ satisfying $\|X\|_{L^{p}}:=\mathrm{E}\left[|X|^{p}\right]^{1 / p}<\infty$. The norm $|\cdot|$ represents the canonic norm on $\mathbb{R}^{d}$ or on $\mathbb{M}^{d}$ and $\langle\cdot, \cdot\rangle$ denotes the usual scalar product on $\mathbb{R}^{d}$. For a function $f \in C^{1}, \nabla_{x} f$ denotes the Jacobian matrix of $f$ with respect to $x$. Finally, for ease of notation, we will sometimes write $\mathrm{E}_{s}[\cdot]$ for $\mathrm{E}\left[\cdot \mid \mathcal{F}_{S}\right], s \in[0, T]$.

\section{Discretely reflected BSDE}

\subsection{Definition}

Let $T>0$ be a finite-time horizon, and let $(\Omega, \mathcal{F}, \mathrm{P})$ be a stochastic basis supporting a $d$-dimensional Brownian motion $W$. We assume that the filtration $\mathbb{F}=\left(\mathcal{F}_{t}\right)_{t \leq T}$ generated by $W$ satisfies the usual assumptions and that $\mathcal{F}_{T}=\mathcal{F}$.

Let $X$ be the solution on $[0, T]$ of

$$
X_{t}=X_{0}+\int_{0}^{t} b\left(X_{u}\right) \mathrm{d} u+\int_{0}^{t} \sigma\left(X_{u}\right) \mathrm{d} W_{u},
$$

where $X_{0} \in \mathbb{R}^{d}$, and $b: \mathbb{R}^{d} \mapsto \mathbb{R}^{d}$ and $\sigma: \mathbb{R}^{d} \mapsto \mathbb{M}^{d}$ satisfy one of the following assumptions, for some positive constant $L$ :

(H $x 1) \quad b$ and $\sigma$ are $L$-Lipschitz continuous,

(Hx2) $b$ and $\sigma$ are $C_{b}^{1}$ with $L$-Lipschitz continuous first derivatives bounded by $L$.

Remark 2.1. Observe that, as in [3] and contrary to [15], we make no uniform ellipticity condition on $\sigma$. In particular, the standard results of the partial differential equation literature cannot be used to derive strong regularity properties on the solution of the partial differential equation of the form (1.2) associated to (1.3).

Under ( $\mathrm{H} x 1)$, we clearly have $X \in s^{2}\left(\mathbb{R}^{d}\right)$, where, for $p \geq 1$ and $E=\mathbb{R}^{d}$ or $E=$ $\mathbb{M}^{d}, \varsigma^{p}(E)$ is the set of $E$-valued progressively measurable processes $U$ such that $\|U\|_{\delta^{p}}:=$ $\left\|\sup _{t \in[0, T]} U_{t}\right\|_{L^{p}}<\infty$. In particular,

$$
\|X\|_{\delta^{2}} \leq C_{L},
$$

where, from now on, $C_{L}$ denotes a generic constant whose value may change from line to line, but which depends only on $L, T, X_{0}$, and $d$ (we write $C_{L}^{p}$ if it also depends on some extra parameter $p \geq 1$ ).

We then introduce a family of closed convex domains $\left(\mathcal{O}_{x}\right)_{x \in \mathbb{R}^{d}}$ :

$$
\mathcal{O}_{x}:=\left\{y \in \mathbb{R}^{d} \mid \text { for all } \ell \in\{1, \ldots, d\}, l^{\ell}(x) \leq y^{\ell} \leq h^{\ell}(x)\right\},
$$


where the maps $h, l: \mathbb{R}^{d} \rightarrow \mathbb{R}^{d}$ satisfy one of the following regularity assumptions.

(Hb1) $h$ and $l$ are $L$-Lipschitz continuous.

(Hb2) For each $\ell \in\{1, \ldots, d\}, h^{\ell}$ and $l^{\ell}$ verify, for some $\left(\rho_{1}^{\ell}, \rho_{2}^{\ell}\right): \mathbb{R}^{d} \rightarrow \mathbb{R}^{d} \times \mathbb{R}^{d}, \rho_{3}^{\ell}$ : $\mathbb{R}^{d} \rightarrow \mathbb{R}^{+}$

$$
\begin{gathered}
\left|\rho_{1}^{\ell}(x)\right|+\left|\rho_{2}^{\ell}(x)\right|+\left|\rho_{3}^{\ell}(x)\right| \leq L\left(1+|x|^{L}\right), \\
l^{\ell}(x)-l^{\ell}(y) \leq \rho_{1}^{\ell}(x)^{\top}(y-x)+\rho_{3}^{\ell}(x)|x-y|^{2} \quad \text { for all } x, y \in \mathbb{R}^{d}, \\
h^{\ell}(y)-h^{\ell}(x) \leq \rho_{2}^{\ell}(x)^{\top}(y-x)+\rho_{3}^{\ell}(x)|x-y|^{2} \quad \text { for all } x, y \in \mathbb{R}^{d} .
\end{gathered}
$$

This assumption is slightly weaker than the semi-convexity assumption of [2, Definition 1].

(Hb3) $h$ and $l$ are $C_{b}^{2}$ with $L$-Lipschitz continuous first and second derivatives bounded by $L$ and there is a $\varepsilon \in\left(L^{-1}, \infty\right)$ such that $h^{\ell}>l^{\ell}+\varepsilon$ for each $\ell \in\{1, \ldots, d\}$.

Observe that $(\mathrm{H} b 3)$ implies $(\mathrm{H} b 2)$, which in turn implies $(\mathrm{H} b 1)$.

Given a set of reflection times

$$
\Re:=\left\{0=: r_{0}<r_{1}<\cdots<r_{\kappa-1}<r_{\kappa}:=T\right\}, \quad \kappa \geq 1,
$$

the solution of the discretely reflected BSDE is a triplet $\left(Y^{\mathfrak{R}}, \tilde{Y}^{\mathfrak{R}}, Z^{\mathfrak{R}}\right)$ satisfying

$$
Y_{T}^{\Re}=\tilde{Y}_{T}^{\Re}:=g\left(X_{T}\right) \in \mathcal{O}_{X_{T}},
$$

and, for $j \leq \kappa-1$ and $t \in\left[r_{j}, r_{j+1}\right)$,

$$
\begin{gathered}
\tilde{Y}_{t}^{\Re}=Y_{r_{j+1}}^{\Re}+\int_{t}^{r_{j+1}} f\left(\Theta_{u}^{\Re}\right) \mathrm{d} u-\int_{t}^{r_{j+1}}\left(Z_{u}^{\Re}\right)^{\top} \mathrm{d} W_{u}, \\
Y_{t}^{\Re}=\mathcal{R}\left(t, X_{t}, \tilde{Y}_{t}^{\Re}\right),
\end{gathered}
$$

with $\Theta^{\Re}=\left(X, \tilde{Y}^{\Re}, Z^{\Re}\right)$.

Here, $g: \mathbb{R}^{d} \mapsto \mathbb{R}^{d}$ and $f: \mathbb{R}^{d} \times \mathbb{R}^{d} \times \mathbb{M}^{d} \mapsto \mathbb{R}^{d}$ are $L$-Lipschitz continuous and

$$
\mathcal{R}^{\ell}(t, x, y):=y^{\ell}+\left(\left[l^{\ell}(x)-y^{\ell}\right]^{+}-\left[y^{\ell}-h^{\ell}(x)\right]^{+}\right) \mathbf{1}_{\{t \in \mathfrak{R}\}}
$$

for $(t, x, y) \in[0, T] \times \mathbb{R}^{d} \times \mathbb{R}$ and $\ell \in\{1, \ldots, d\}$.

Observe that

$$
Y_{t}^{\Re}=\tilde{Y}_{t}^{\Re} \quad \text { for } t \notin \Re \backslash\{T\} .
$$

Remark 2.2. Under $(\mathrm{H} x 1)$ and $(\mathrm{H} b 1)$, such a solution can be defined by backward induction. At each step the existence and uniqueness in $8^{2}\left(\mathbb{R}^{d}\right) \times \mathscr{H}^{2}\left(\mathbb{M}^{d}\right)$ follow from, e.g. [8]. Here, for $p \geq 1$ and $E=\mathbb{M}^{d}$ or $E=\mathbb{M}^{d^{2}, d}, \mathscr{H}^{p}(E)$ is the set of progressively measurable $E$-valued processes $V$ satisfying

$$
\|V\|_{\mathscr{H}^{p}}:=\left\|\left(\int_{0}^{T}\left|V_{r}\right|^{2} \mathrm{~d} r\right)^{1 / 2}\right\|_{L^{p}}<\infty .
$$

Remark 2.3. (i) The case where $\left(Y^{\Re}, X\right)$ takes values in $\mathbb{R}^{n} \times \mathbb{R}^{d}$ with $n \neq d$ can be treated in our framework. Indeed, if $d<n$, we can set $X^{i}:=0$, i.e. $b^{i}=0, \sigma^{i}=0$, and $X_{0}^{i}=0$, for $i>d$. Recall that we make no ellipticity assumption. If $d>n$, we can set $g^{i}=f^{i}:=0$, which implies that $Y^{i}=0$ for $i>n$, and work with $\mathcal{O}_{x} \times[-\varepsilon, \varepsilon]^{d-n}, \varepsilon>0, x \in \mathbb{R}^{d}$. 
(ii) In view of Remark 2.1, it is clear that the results hold for nonhomogeneous SDEs: it suffices to consider $(t, X)$ as the forward diffusion.

In Appendix A we provide useful a priori estimates for 'reflected' BSDEs in a somehow abstract setting. In our framework, they read as follows (recall that $(\kappa+1)$ is the number of time steps in the reflection grid $\mathfrak{R}$ ).

Proposition 2.1. Under (Hxl) and (Hbl), the following holds

$$
\sup _{t \in[0, T]} \mathrm{E}\left[\left|\tilde{Y}_{t}^{\Re}\right|^{2}+\left|Y_{t}^{\Re}\right|^{2}\right]+\left\|Z^{\Re}\right\|_{\mathcal{H}^{2}}^{2} \leq C_{L} \kappa .
$$

Moreover, if

$(\mathrm{H} f) f^{\ell}$ depends on $z^{. i}$ only for $i=\ell$ (i.e. $\nabla_{z^{i}} f^{\ell} \mathbf{1}_{\{i \neq \ell\}}=0$ if $f \in C^{1}$ )

holds, then

$$
\sup _{t \in[0, T]} \mathrm{E}\left[\left|\tilde{Y}_{t}^{\Re}\right|^{2}+\left|Y_{t}^{\Re}\right|^{2}\right] \leq C_{L}
$$

Proof. It suffices to apply Proposition A.1 in Appendix A, with $\eta_{r}=\left|X_{r}\right|$ and $\xi_{r}=\left|h\left(X_{r}\right)\right| \mathrm{V}$ $\left|l\left(X_{r}\right)\right|, r \in \Re$, and recalling (2.1).

\subsection{Dependence on the parameters}

We now present some estimates on the variation in the solution of (2.2) induced by a variation in the data. Later on, this will allow us to work with smooth parameters $(f, g$, etc.) before turning to the general case by an approximation argument (see, e.g. Proposition 4.2, below).

In the rest of this section we consider two discretely reflected BSDEs constructed as follows.

For $i \in\{1,2\}$, let $X^{i}$ be an element of $\delta^{2}\left(\mathbb{R}^{d}\right)$, let $f_{i}$ and $g_{i}$ be $L$-Lipschitz continuous functions, and let the $h_{i}$ and $l_{i}$ boundaries satisfy $(\mathrm{H} b 1)$. We denote by $\left(Y^{\mathfrak{R}, i}, \tilde{Y}^{\mathfrak{R}, i}, Z^{\mathfrak{R}, i}\right)$ the solutions of the discretely reflected BSDE associated to these two sets of data and $\Theta^{\Re, i}:=\left(X^{i}\right.$, $\left.\tilde{Y}^{\Re, i}, Z^{\mathfrak{R}, i}\right)$. We then define $\delta Y^{\mathfrak{R}}:=Y^{\mathfrak{R}, 1}-Y^{\mathfrak{R}, 2}, \delta \tilde{Y}^{\Re}:=\tilde{Y}^{\mathfrak{R}, 1}-\tilde{Y}^{\mathfrak{R}, 2}, \delta Z^{\mathfrak{R}}:=Z^{\mathfrak{R}, 1}$ $Z^{\Re, 2}$, and $\delta X:=X^{1}-X^{2}, \delta f:=f_{1}\left(\Theta^{\Re, 1}\right)-f_{2}\left(\Theta^{\Re, 1}\right), \delta g:=g_{1}\left(X^{1}\right)-g_{2}\left(X^{1}\right), \delta h:=$ $h_{1}\left(X^{1}\right)-h_{2}\left(X^{1}\right)$, and $\delta l:=l_{1}\left(X^{1}\right)-l_{2}\left(X^{1}\right)$.

Proposition 2.2. Under $(H x)$ and (Hbl), the following holds:

$$
\sup _{t \in[0, T]} \mathrm{E}\left[\left|\delta Y_{t}^{\Re \mathfrak{R}}\right|^{2}\right]+\|\delta Z\|_{\mathscr{H}^{2}}^{2} \leq C_{L}\left(\kappa \mathrm{E}\left[\max _{r \in \mathfrak{R}}\left(\left|\delta X_{r}\right|^{2}+\left|\delta h_{r}\right|^{2}+\left|\delta l_{r}\right|^{2}\right)\right]+\|\delta f\|_{\mathscr{H}^{2}}^{2}+\left\|\delta g_{T}\right\|_{L^{2}}^{2}\right) .
$$

The proof of this result requires the following lemma whose proof uses a key argument which will be very important below when studying the convergence of the Euler scheme type approximation of (2.2).

Lemma 2.1. Let (Hx l) and (Hbl) hold. Then, for each $r \in \mathfrak{R} \backslash\{T\}$ and $\ell \in\{1, \ldots, d\}$, there exists $S_{r}^{\ell}$ and $Q_{r}^{\ell}$ in $\mathcal{F}_{r}$ such that $S_{r}^{\ell} \cap Q_{r}^{\ell}=\varnothing$ and

$$
\begin{aligned}
\left|\left(Y_{r}^{\Re, 1}\right)^{\ell}-\left(Y_{r}^{\Re, 2}\right)^{\ell}\right| \leq & \left|\left(\tilde{Y}_{r}^{\Re, 1}\right)^{\ell}-\left(\tilde{Y}_{r}^{\Re, 2}\right)^{\ell}\right| \mathbf{1}_{S_{r}^{\ell}} \\
& +\left(\left|l_{1}^{\ell}\left(X_{r}^{1}\right)-l_{2}^{\ell}\left(X_{r}^{2}\right)\right|+\left|h_{1}^{\ell}\left(X_{r}^{1}\right)-h_{2}^{\ell}\left(X_{r}^{2}\right)\right|\right) \mathbf{1}_{Q_{r}^{\ell} .}
\end{aligned}
$$

Proof. For ease of notation, we work with $d=1$ and omit the exponent $\ell$. Appropriate $S_{r}$ and $Q_{r}$ are constructed by considering different disjoint cases, depending on the position of $\tilde{Y}_{r}^{\Re, 1}$ and $\tilde{Y}_{r}^{\Re, 2}$. 
Case 1. On $\left\{l_{1}\left(X_{r}^{1}\right)<\tilde{Y}_{r}^{\Re, 1}<h_{1}\left(X_{r}^{1}\right)\right\}$, three different cases may occur depending on the position of $\tilde{Y}^{\Re, 2}$.

(i) On $\left\{l_{2}\left(X_{r}^{2}\right)<\tilde{Y}_{r}^{\Re, 2}<h_{2}\left(X_{r}^{2}\right)\right\}$, we have $Y_{r}^{\Re, 1}-Y_{r}^{\Re, 2}=\tilde{Y}_{r}^{\Re, 1}-\tilde{Y}_{r}^{\Re, 2}$.

(ii) On $\left\{\tilde{Y}_{r}^{\Re, 2} \leq l_{2}\left(X_{r}^{2}\right)\right\}$, we have $Y_{r}^{\Re, 2}=\mathcal{P}\left(X_{r}^{2}, \tilde{Y}_{r}^{\Re, 2}\right)=l_{2}\left(X_{r}^{2}\right)$. If $l_{2}\left(X_{r}^{2}\right) \leq \tilde{Y}_{r}^{\Re, 1}$ then $0 \leq Y_{r}^{\Re, 1}-Y_{r}^{\Re, 2}=\tilde{Y}_{r}^{\Re, 1}-l_{2}\left(X_{r}^{2}\right) \leq \tilde{Y}_{r}^{\Re, 1}-\tilde{Y}_{r}^{\Re, 2}$. If $l_{2}\left(X_{r}^{2}\right)>\tilde{Y}_{r}^{\Re, 1}$ then $0 \leq$ $l_{2}\left(X_{r}^{2}\right)-\tilde{Y}_{r}^{\Re, 1}=Y_{r}^{\Re, 2}-Y_{r}^{\Re, 1} \leq l_{2}\left(X_{r}^{2}\right)-l_{1}\left(X_{r}^{1}\right)$.

(iii) On $\left\{h_{2}\left(X_{r}^{2}\right) \leq \tilde{Y}_{r}^{\Re, 2}\right\}$, similar arguments based on the comparison between $h_{2}\left(X_{r}^{2}\right)$ and $\tilde{Y}_{r}^{\Re, 1}$ lead to $\left|Y_{r}^{\Re, 1}-Y_{r}^{\Re, 2}\right| \leq\left|\tilde{Y}_{r}^{\Re, 1}-\tilde{Y}_{r}^{\Re, 2}\right|$ on $\left\{\tilde{Y}_{r}^{\Re, 1} \leq h_{2}\left(X_{r}^{2}\right)\right\}$ and $\left|Y_{r}^{\Re, 1}-Y_{r}^{\Re, 2}\right| \leq$ $\left|h_{2}\left(X_{r}^{2}\right)-h_{1}\left(X_{r}^{1}\right)\right|$ on $\left\{h_{2}\left(X_{r}^{2}\right)<\tilde{Y}_{r}^{\Re, 1}\right\}$.

Case 2. We now study the case $\left\{\tilde{Y}_{r}^{\Re, 1} \leq l_{1}\left(X_{r}^{1}\right)\right\}$, which implies that $Y_{r}^{\Re, 1}=l_{1}\left(X_{r}^{1}\right)$.

(i) On $\left\{\tilde{Y}_{r}^{\Re, 2} \leq l_{2}\left(X_{r}^{2}\right)\right\}$, we have $Y_{r}^{\Re, 1}-Y_{r}^{\Re, 2}=l_{1}\left(X_{r}^{1}\right)-l_{2}\left(X_{r}^{2}\right)$.

(ii) On $\left\{l_{2}\left(X_{r}^{2}\right)<\tilde{Y}_{r}^{\Re, 2}<h_{2}\left(X_{r}^{2}\right)\right\}$, there are two disjoint cases. On $\left\{Y_{r}^{\Re, 2}<Y_{r}^{\Re, 1}\right\}, 0 \leq$ $Y_{r}^{\Re, 1}-Y_{r}^{\mathfrak{R}, 2} \leq l_{1}\left(X_{r}^{1}\right)-l_{2}\left(X_{r}^{2}\right)$. On $\left\{Y_{r}^{\mathfrak{R}, 2} \geq Y_{r}^{\mathfrak{R}, 1}\right\}, 0 \leq Y_{r}^{\mathfrak{R}, 2}-Y_{r}^{\mathfrak{R}, 1} \leq \tilde{Y}_{r}^{\mathfrak{R}, 2}-\tilde{Y}_{r}^{\mathfrak{R}, 1}$.

(iii) Finally, on $\left\{\tilde{Y}_{r}^{\Re, 2} \geq h_{2}\left(X_{r}^{2}\right)\right\}$ we also have two disjoint cases. On $\left\{h_{2}\left(X_{r}^{2}\right)>Y_{r}^{\Re, 1}\right\}$, $0 \leq Y_{r}^{\Re, 2}-Y_{r}^{\Re, 1} \leq \tilde{Y}_{r}^{\Re, 2}-\tilde{Y}_{r}^{\mathfrak{R}, 1}$. On $\left\{h_{2}\left(X_{r}^{2}\right) \leq Y_{r}^{\mathfrak{R}, 1}\right\}, 0 \leq Y_{r}^{\mathfrak{R}, 1}-h_{2}\left(X_{r}^{2}\right) \leq$ $h_{1}\left(X_{r}^{1}\right)-h_{2}\left(X_{r}^{2}\right)$.

Case 3 . By symmetry, the case $\tilde{Y}_{r}^{\Re, 1} \geq h_{1}\left(X_{r}^{1}\right)$ is handled similarly.

Proof of Proposition 2.2. The proof of this proposition relies on the abstract results of Proposition A.1 in Appendix A. For $t \in\left[r_{j}, r_{j+1}\right)$, we have

$$
\delta \tilde{Y}_{t}^{\Re}=\delta Y_{r_{j+1}}^{\Re}+\int_{t}^{r_{j+1}} \hat{f}(u) \mathrm{d} u-\int_{t}^{r_{j+1}}\left(\delta Z_{u}^{\Re}\right)^{\top} \mathrm{d} W_{u},
$$

where $\hat{f}:=\delta f+f_{2}\left(\Theta^{\Re, 1}\right)-f_{2}\left(\Theta^{\Re, 2}\right)$.

Since $f_{2}$ is $L$-Lipschitz continuous, we have

$$
|\hat{f}(u)|^{2} \leq C_{L}\left(\left|\eta_{u}\right|^{2}+\left|\delta \tilde{Y}_{u}^{\Re}\right|^{2}+\left|\delta Z_{u}^{\Re}\right|^{2}\right) \quad \text { with } \quad \eta_{u}:=\left|\delta f_{u}\right|+|\delta X| .
$$

Moreover, using Lemma 2.1, we can set $\xi:=2 L|\delta X|+|\delta l|+|\delta h|$, since $h_{2}$ and $l_{2}$ are $L$-Lipschitz continuous.

The proof is then concluded by appealing to Proposition A.1 and observing that $\left|\delta Y_{T}^{\Re}\right| \leq$ $L\left|\delta X_{T}\right|+\left|\delta g_{T}\right|$, since $g_{2}$ is $L$-Lipschitz continuous.

Remark 2.4. As mentioned in the introduction, when $h$ and $l$ are constant, the results of this paper are a subcase of the results given in [6, Chapter 3], in which the case of a general fixed convex domain $\mathcal{O}$ is considered. The case of a general convex domain evolving randomly in time cannot be treated in our framework. This is due to the fact that we cannot retrieve the results of Lemma 2.1, in general. The question of the approximation of the solution of reflected BSDEs in this general case is left for further research. 


\section{Representation results for $Z^{\mathfrak{R}}$}

In this section we provide different representations for $Z^{\Re}$. The first two representations are stated in terms of the Malliavin derivatives of $\left(X, Y^{\mathfrak{R}}, Z^{\mathfrak{R}}\right)$, the last representation is based on their associated 'first variation' processes.

In order to ensure that $\left(X, Y^{\Re}, Z^{\Re}\right)$ are 'smooth' enough, we will work under the additional assumption that

$(\mathrm{H} r) h, l, f, b$, and $\sigma$ are $C_{b}^{1}$.

These representations will allow us to provide regularity results for $\left(Y^{\Re}, Z^{\mathfrak{R}}\right)$ under $(\mathrm{H} r)$. This assumption will then be relaxed by using an approximation argument based on Proposition 2.2.

\subsection{Malliavin differentiability of $\left(X, Y^{\Re}, Z^{\mathfrak{R}}\right)$}

In the sequel we denote by $\mathbb{D}^{1,2}$ the space of random variables $F$ which are differentiable in the Malliavin sense and such that

$$
\|F\|_{L^{2}}^{2}+\int_{0}^{T}\left\|D_{t} F\right\|_{L^{2}}^{2} \mathrm{~d} t<\infty .
$$

Here, $D_{t} F$ denotes the Malliavin derivative of $F$ at time $t \leq T$; see, e.g. [16].

We also consider the space $\mathbb{L}^{1,2}$ of adapted processes $V$ such that, after possibly passing to a suitable version, $V_{s} \in \mathbb{D}^{1,2}$ for all $s \leq T$ and

$$
\|V\|_{\mathscr{H}^{2}}+\int_{0}^{T}\left\|D_{t} V\right\|_{\mathscr{H}^{2}} \mathrm{~d} t<\infty .
$$

In the following we will always work with a suitable version if necessary.

Under $(\mathrm{H} r), X$ belongs to $\mathbb{L}^{1,2}$; see [16]. It follows that $\mathcal{R}^{\ell}(r, X, F) \in \mathbb{D}^{1,2}$ whenever $F \in \mathbb{D}^{1,2}$ and

$D_{t} \mathcal{R}^{\ell}(r, X, F)=D_{t} F^{\ell}+\left(D_{t} l^{\ell}\left(X_{r}\right)-D_{t} F^{\ell}\right) \mathbf{1}_{\left\{l^{\ell}\left(X_{r}\right)>F^{\ell}\right\}}-\left(D_{t} F^{\ell}-D_{t} h^{\ell}\left(X_{r}\right)\right) \mathbf{1}_{\left\{h^{\ell}\left(X_{r}\right)<F^{\ell}\right\}}$.

Indeed, by a direct adaptation of the proof of Proposition 1.2.3 of [16] we deduce that, for $G \in \mathbb{D}^{1,2},[G]^{+}$belongs to $\mathbb{D}^{1,2}$ and $D_{t}[G]^{+}=\alpha\left(D_{t} G\right)$, where $\alpha$ is a random variable bounded by 1 satisfying $\mathbf{1}_{\{G>0\}} \alpha=\mathbf{1}_{\{G>0\}}$. Thus, Proposition 1.3.7 of [16] implies that $D_{t}[G]^{+}=D_{t} G \mathbf{1}_{\{G>0\}}$ if $G \in \mathbb{D}^{1,2}$.

Combining (2.2), (3.1), and Proposition 5.3 of [8] with an induction argument, we find that $\left(\tilde{Y}^{\Re}, Z^{\mathfrak{R}}\right)$ belongs to $\mathbb{L}^{1,2}$ and that a version of $D_{t}\left(\left(\tilde{Y}^{\mathfrak{R}}\right)^{\ell},\left(Z^{\mathfrak{R}}\right)^{\cdot \ell}\right)$ is given by the solution in $\S^{2}\left(\mathbb{R}^{d}\right) \times \mathscr{H}^{2}\left(\mathbb{M}^{d}\right)$ of

$$
\begin{aligned}
D_{t}\left(\tilde{Y}_{s}^{\Re}\right)^{\ell}= & D_{t}\left(Y_{r_{j+1}}^{\Re}\right)^{\ell}+\int_{s}^{r_{j+1}}\left(\nabla_{x} f^{\ell}\left(\Theta_{u}^{\Re}\right) D_{t} X_{u}+\nabla_{y} f^{\ell}\left(\Theta_{u}^{\Re}\right) D_{t} \tilde{Y}_{u}^{\Re}\right) \mathrm{d} u \\
& +\int_{s}^{r_{j+1}} \sum_{i=1}^{d} \nabla_{z^{i}} f^{\ell}\left(\Theta_{u}^{\Re}\right) D_{t}\left(Z_{u}^{\Re}\right)^{\cdot i} \mathrm{~d} u-\int_{s}^{r_{j+1}} \sum_{k=1}^{d} D_{t}\left(Z_{u}^{\Re}\right)^{k \ell} \mathrm{d} W_{u}^{k}
\end{aligned}
$$

for $s \in\left[r_{j}, r_{j+1}\right), j<\kappa$, with the terminal condition

$$
D_{t}\left(\tilde{Y}_{T}^{\Re}\right)^{\ell}=\nabla g^{\ell}\left(X_{T}\right) D_{t} X_{T} .
$$

We conclude this section with some a priori estimates that will be used later on. The first one concerning $D X$ is standard and, therefore, we omit the proof (see, e.g. [16]). 
Proposition 3.1. Let $(\mathrm{Hr})$ hold. Then, for all $p \geq 2$,

$$
\sup _{s \leq u \wedge t}\left\|D_{s} X_{t}-D_{s} X_{u}\right\|_{L^{p}}+\left\|\left(D_{t} X-D_{u} X\right) \mathbf{1}_{[t \vee u, T]}\right\|_{s^{p}} \leq C_{L}^{p}|t-u|^{1 / 2}, \quad t, u \leq T,
$$

and

$$
\left\|\sup _{s \leq T}\left|D_{s} X\right|\right\|_{s p} \leq C_{L}^{p}
$$

We now turn to the study of $\left(D Y^{\Re}, D Z^{\Re}\right)$. For ease of notation, we will from now on denote by $\beta$ an $\mathcal{F}_{T}$-measurable positive random variable whose value may change from line to line, but satisfies $\mathrm{E}\left[\beta^{p}\right] \leq C_{L}^{p}$ for all $p \geq 1$.

Proposition 3.2. Let $(\mathrm{Hr})$ hold. Then, for $s \leq t \leq T$,

$$
\left|D_{s} \tilde{Y}_{t}^{\Re}\right|^{2}+\left|D_{s} Y_{t}^{\Re}\right|^{2}+\mathrm{E}_{t}\left[\int_{t}^{T} \sum_{\ell=1}^{d}\left|D_{s}\left(Z_{u}^{\Re}\right)^{\cdot \ell}\right|^{2} \mathrm{~d} u\right] \leq \kappa \mathrm{E}_{t}[\beta] .
$$

If $(H f)$ holds then, for $p \geq 2$,

$$
\left|D_{s} \tilde{Y}_{t}^{\Re}\right|^{p}+\left|D_{s} Y_{t}^{\Re}\right|^{p}+\mathrm{E}_{t}\left[\sum_{\ell=1}^{d} \int_{t}^{\tau_{j}^{\ell}}\left|D_{s}\left(Z_{u}^{\Re}\right)^{\ell \ell}\right|^{2} \mathrm{~d} u\right] \leq \mathrm{E}_{t}[\beta]
$$

and

$$
\left|D_{s} \tilde{Y}_{t}^{\Re}\right|^{p}+\left|D_{s} Y_{t}^{\Re}\right|^{p} \leq \mathrm{E}_{t}[\beta]
$$

for $j \leq \kappa-1, t \in\left[r_{j}, r_{j+1}\right)$, and $s \leq t$, where

$$
\tau_{j}^{\ell}=\inf \left\{t \in \Re \mid t \geq r_{j+1},\left(\tilde{Y}_{t}^{\Re}\right)^{\ell} \notin\left[l^{\ell}\left(X_{t}\right), h^{\ell}\left(X_{t}\right)\right]\right\} \wedge T, \quad j \leq \kappa-1, \ell \leq d .
$$

Proof. Recall that, for $F \in \mathbb{D}^{1,2}, D F=\left(D^{1} F, \ldots, D^{d} F\right)$, where $D^{i}$ denotes the Malliavin derivatives with respect to $W^{i}$. Fix $q \in\{1, \ldots, d\}$. By (3.2), we have, for all $t \leq s \in\left[r_{j}, r_{j+1}\right)$ and $j<\kappa$,

$$
\begin{aligned}
D_{t}^{q} \tilde{Y}_{s}^{\Re}= & D_{t}^{q} Y_{r_{j+1}}^{\Re}-\int_{s}^{r_{j+1}}\left(D_{t}^{q} Z_{u}^{\Re}\right)^{\top} \mathrm{d} W_{u} \\
& +\int_{s}^{r_{j+1}}\left(\nabla_{x} f\left(\Theta_{u}^{\Re}\right) D_{t}^{q} X_{u}+\nabla_{y} f\left(\Theta_{u}^{\Re}\right) D_{t}^{q} \tilde{Y}_{u}^{\Re}+\nabla_{z} f\left(\Theta_{u}^{\Re}\right) D_{t}^{q} Z_{u}^{\Re}\right) \mathrm{d} u .
\end{aligned}
$$

Since $f$ is $C_{b}^{1}$ under (Hr), (A.2) in Appendix A holds with $\eta=\left|D_{t}^{q} X\right|$. Clearly, (A $f$ ) in Appendix A holds under $(\mathrm{H} f)$.

Moreover, it follows from (3.1) that $\left(D_{t}^{q} Y^{\Re}, D_{t}^{q} \tilde{Y}^{\Re}\right)$ satisfies (A0) in Appendix A (take $S_{r}^{\ell}=\left\{\left(Y_{r}^{\Re}\right)^{\ell} \in\left[l^{\ell}\left(X_{r}\right), h^{\ell}\left(X_{r}\right)\right]\right\}, \xi_{r}^{\ell}=C\left|D_{t}^{q} X_{r}\right|$, for $r \in \Re$ and $\left.\ell \in\{1, \ldots, d\}\right)$.

The result is then a direct application of Proposition A.1 and Corollary A.1.

Similar arguments based on Proposition A.1 also lead to the following result.

Proposition 3.3. Under $(\mathrm{Hr})$, we have, for all $t \leq T$ and $r, s \leq t$,

$$
\begin{aligned}
& \left|D_{s} \tilde{Y}_{t}^{\Re}-D_{r} \tilde{Y}_{t}^{\Re}\right|^{2}+\left|D_{s} Y_{t}^{\Re}-D_{r} Y_{t}^{\Re}\right|^{2}+\mathrm{E}_{t}\left[\int_{t}^{T} \sum_{\ell=1}^{d}\left|D_{s}\left(Z_{u}^{\Re}\right)^{\ell \ell}-D_{r}\left(Z_{u}^{\Re}\right)^{\cdot \ell}\right|^{2} \mathrm{~d} u\right] \\
& \quad \leq \kappa \mathrm{E}_{t}[\beta]|s-r| .
\end{aligned}
$$


$\operatorname{Under}(H f)$,

$$
\begin{aligned}
& \left|D_{s} \tilde{Y}_{t}^{\Re}-D_{r} \tilde{Y}_{t}^{\Re}\right|^{2}+\left|D_{s} Y_{t}-D_{r} Y_{t}\right|^{2}+\mathrm{E}_{t}\left[\sum_{\ell=1}^{d} \int_{t}^{\tau_{j}^{\ell}}\left|D_{s}\left(Z_{u}^{\Re}\right)^{\cdot \ell}-D_{r}\left(Z_{u}^{\Re}\right)^{\cdot \ell}\right|^{2} \mathrm{~d} u\right] \\
& \quad \leq \mathrm{E}_{t}[\beta]|s-r|
\end{aligned}
$$

for $j \leq \kappa-1, t \in\left[r_{j}, r_{j+1}\right)$, and $r, s \leq t$.

\subsection{Representation in terms of Malliavin derivatives of $\left(X, Y^{\mathfrak{R}}, Z^{\mathfrak{R}}\right)$}

It follows from [8, Proposition 5.3] and (2.2) that $\left(D_{t} Y_{t}^{\Re}\right)_{t \leq T}$ is a version of $Z^{\Re}$. Hence, (3.2) implies that $Z^{\mathfrak{R}}$ admits a version satisfying

$$
\begin{array}{r}
\left(Z_{t}^{\mathfrak{R}}\right)^{\top}=\mathrm{E}_{t}\left[D_{t} Y_{r_{j+1}}^{\Re}+\int_{t}^{r_{j+1}}\left(\nabla_{x} f\left(\Theta_{u}^{\Re}\right) D_{t} X_{u}+\nabla_{y} f\left(\Theta_{u}^{\Re}\right) D_{t} \tilde{Y}_{u}^{\Re}\right.\right. \\
\left.\left.+\sum_{i=1}^{d} \nabla_{z^{\prime i}} f\left(\Theta_{u}^{\Re}\right) D_{t}\left(Z_{u}^{\mathfrak{R}}\right)^{\cdot i}\right) \mathrm{~d} u\right]
\end{array}
$$

for each $j \leq \kappa-1$ and $t \in\left[r_{j}, r_{j+1}\right)$.

Following the arguments of [3], we can get rid of the term $D_{t} Y_{r_{j+1}}^{\Re}$ in the above expression.

Corollary 3.1. Let $(H r)$ hold. Then, for each $\ell \in\{1, \ldots, d\}$, there is a version of $\left(Z^{\mathfrak{R}}\right)^{\cdot \ell}$ such that, for each $j \leq \kappa-1$ and $s \leq t \in\left[r_{j}, r_{j+1}\right)$,

$$
\begin{aligned}
\left(\left(Z_{t}^{\mathfrak{R}}\right)^{\ell}\right)^{\top}=\mathrm{E}_{t}[ & \nabla \phi_{\tau_{j}^{\ell}}^{\ell}\left(D_{t} X\right)_{\tau_{j}^{\ell}}+\int_{t}^{\tau_{j}^{\ell}} \nabla_{x} f^{\ell}\left(\Theta_{u}^{\Re}\right) D_{t} X_{u} \mathrm{~d} u \\
& \left.+\int_{t}^{\tau_{j}^{\ell}}\left(\nabla_{y} f^{\ell}\left(\Theta_{u}^{\Re}\right) D_{t} \tilde{Y}_{u}^{\Re}+\sum_{i=1}^{d} \nabla_{z^{\cdot i}} f^{\ell}\left(\Theta_{u}^{\Re}\right) D_{t}\left(Z_{u}^{\Re}\right)^{\cdot i}\right) \mathrm{d} u\right],
\end{aligned}
$$

where, for $r \in \Re$,

$$
\nabla \phi_{r}^{\ell}:=\nabla g^{\ell}\left(X_{r}\right) \mathbf{1}_{\{r=T\}}+\left(\nabla l^{\ell}\left(X_{r}\right) \mathbf{1}_{\left\{l^{\ell}\left(X_{r}\right)>\left(\tilde{Y}_{r}^{\Re}\right)^{\ell}\right\}}+\nabla h^{\ell}\left(X_{r}\right) \mathbf{1}_{\left\{h^{\ell}\left(X_{r}\right)<\left(\tilde{Y}_{r}^{\Re}\right)^{\ell}\right\}}\right) \mathbf{1}_{\{r<T\}} .
$$

Proof. For $\ell \in\{1, \ldots, d\}$ and $q \leq \kappa-1$, we denote by $\xi_{q}^{\ell}$ the random index such that $r_{\xi_{q}^{\ell}}=\tau_{q}^{\ell}$ (recall the definition of $\tau_{q}^{\ell}$ in Proposition 3.2). On $\left\{\tau_{q}^{\ell}=r_{q+1}\right\}$, the result is obvious. On $\left\{\tau_{q}^{\ell}>r_{q+1}\right\}$, summing up from $q$ to $\xi_{q}^{\ell}$ in (3.2) applied to $s=r_{q+1}$ and using (3.1) leads to

$$
\begin{aligned}
D_{t}\left(\tilde{Y}_{s}^{\Re}\right)^{\ell}= & \nabla \phi_{\tau_{j}^{\ell}}^{\ell}\left(D_{t} X\right)_{\tau_{q}^{\ell}}+\int_{s}^{\tau_{q}^{\ell}}\left(\nabla_{x} f^{\ell}\left(\Theta_{u}^{\Re}\right) D_{t} \chi_{u}+\nabla_{y} f^{\ell}\left(\Theta_{u}^{\Re}\right) D_{t} \tilde{Y}_{u}^{\Re}\right) \mathrm{d} u \\
& +\int_{s}^{\tau_{q}^{\ell}} \sum_{i=1}^{d} \nabla_{z^{\cdot}} f^{\ell}\left(\Theta_{u}^{\Re}\right) D_{t}\left(Z_{u}^{\Re}\right)^{\cdot i} \mathrm{~d} u \\
& -\int_{s}^{\tau_{q}^{\ell}} \sum_{k=1}^{d} D_{t}\left(Z_{u}^{\Re}\right)^{k \ell} \mathrm{d} W_{u}^{k} \quad \text { for } t \leq s \in\left[r_{q}, r_{q+1}\right) .
\end{aligned}
$$

Since $\left(D_{t}\left(\tilde{Y}_{t}^{\Re}\right)^{\ell}\right)_{t \leq T}$ is a version of $\left(\left(Z_{t}^{\Re}\right)^{\cdot \ell}\right)_{t \leq T}$, the required result is obtained by taking the conditional expectation in the above expression. 
Under $(\mathrm{H} f)$, we can also get rid of the term $D_{t} Z^{\Re}$ in expressions (3.4) and (3.5) by arguing as in [3] and [17]. Indeed, applying Itô's lemma to $Y^{\Re} \Lambda^{\ell}$ with

$$
\Lambda_{t}^{\ell}:=\exp \left\{\int_{0}^{t} \nabla_{z^{\cdot \ell}} f^{\ell}\left(\Theta_{u}^{\Re}\right)^{\top} \mathrm{d} W_{u}-\int_{0}^{t} \frac{1}{2}\left|\nabla_{z^{\ell}} f^{\ell}\left(\Theta_{u}^{\Re}\right)\right|^{2} \mathrm{~d} u\right\}, \quad t \leq T,
$$

we directly deduce, from (3.6), the following alternative representation.

Corollary 3.2. Let $(H f)$ and $(H r)$ hold. Then, there is a version of $\left(Z^{\mathfrak{R}}\right)^{\cdot \ell}$ such that

$$
\begin{aligned}
\left(\left(Z_{t}^{\Re}\right)^{\ell \ell}\right)^{\top}=\left(\Lambda_{t}^{\ell}\right)^{-1} \mathrm{E}_{t}\left[\nabla \phi_{\tau_{j}^{\ell}}^{\ell}\left(\Lambda^{\ell} D_{t} X\right)_{\tau_{j}^{\ell}}\right. & +\int_{t}^{\tau_{j}^{\ell}}\left(\nabla_{x} f^{\ell}\left(\Theta_{u}^{\Re}\right)\left(\Lambda^{\ell} D_{t} X\right)_{u}\right. \\
& \left.\left.+\nabla_{y} f^{\ell}\left(\Theta_{u}^{\Re}\right)\left(\Lambda^{\ell} D_{t} \tilde{Y}^{\Re}\right)_{u}\right) \mathrm{~d} u\right]
\end{aligned}
$$

for $s \leq t \in\left[r_{j}, r_{j+1}\right), j \leq \kappa-1$, and $\ell \in\{1, \ldots, d\}$.

Observe that this simplification is no longer possible if $f^{\ell}$ depends of more than one column of $Z^{\Re}$.

Remark 3.1. For later use, observe that

$$
\begin{gathered}
\left\|\sup _{s \leq t \leq T} \Lambda_{t}^{\ell}\right\|_{L^{p}} \leq C_{L}^{p}, \\
\left\|\sup _{u \leq t \wedge s}\left|\Lambda_{t}^{\ell}\left(\Lambda_{u}^{\ell}\right)^{-1}-\Lambda_{s}^{\ell}\left(\Lambda_{u}^{\ell}\right)^{-1}\right|\right\|_{L^{p}} \leq C_{L}^{p}|t-s|^{1 / 2}, \quad t, s \leq T .
\end{gathered}
$$

\subsection{First variation processes associated to $\left(X, Y^{\mathfrak{R}}, Z^{\mathfrak{R}}\right)$}

In Subsection 3.4 below we provide a representation of $Z^{\mathfrak{R}}$ in terms of the first variation process of $\left(X, \tilde{Y}^{\Re}\right)$.

Under $(\mathrm{H} r)$, the first variation process $\nabla X$ of $X$ is well defined and solves, on $[0, T]$,

$$
\nabla X_{t}=I_{d}+\int_{0}^{t} \nabla_{x} b\left(X_{r}\right) \nabla X_{r} \mathrm{~d} r+\int_{0}^{t} \sum_{j=1}^{d} \nabla_{x} \sigma^{\cdot j}\left(X_{r}\right) \nabla X_{r} \mathrm{~d} W_{r}^{j},
$$

where $I_{d}$ is the identity matrix of $\mathbb{M}^{d}$. Its inverse, $(\nabla X)^{-1}$, is the solution on $[0, T]$ of

$$
\begin{aligned}
(\nabla X)_{t}^{-1}=I_{d} & -\int_{0}^{t}(\nabla X)_{r}^{-1}\left(\nabla_{x} b\left(X_{r}\right)-\sum_{j=1}^{d} \nabla_{x} \sigma^{\cdot j}\left(X_{r}\right) \nabla \sigma^{\cdot j}\left(X_{r}\right)\right) \mathrm{d} r \\
& -\int_{0}^{t} \sum_{j=1}^{d}(\nabla X)_{r}^{-1} \nabla_{x} \sigma^{\cdot j}\left(X_{r}\right) \mathrm{d} W_{r}^{j}
\end{aligned}
$$

Recall the well-known relation between $\nabla X$ and $D X$ :

$$
D_{t} X_{s}=\nabla X_{s}\left(\nabla X_{t}\right)^{-1} \sigma\left(X_{t}\right) \mathbf{1}_{t \leq s} \quad \text { for all } t, s \leq T .
$$

Remark 3.2. The following standard estimates hold:

$$
\|\nabla X\|_{\S^{p}}+\left\|(\nabla X)^{-1}\right\|_{\varsigma^{p}} \leq C_{L}^{p} .
$$


Let us now consider the processes $\left(\nabla Y^{\Re}, \nabla \tilde{Y}^{\Re}\right) \in \mathcal{s}^{2}\left(\mathbb{M}^{d}\right) \times \delta^{2}\left(\mathbb{M}^{d}\right)$ and $\nabla Z^{\Re, i} \in$ $\mathscr{H}^{2}\left(\mathbb{M}^{d}\right), i \in\{1, \ldots, d\}$, defined as the solutions of the coupled linear discretely 'reflected' BSDEs:

$$
\nabla Y_{T}^{\Re}=\nabla \tilde{Y}_{T}^{\Re}:=\nabla g\left(X_{T}\right) \nabla X_{T}
$$

and, for $j \leq \kappa-1, t \in\left[r_{j}, r_{j+1}\right)$, and $\ell \in\{1, \ldots, d\}$,

$$
\begin{aligned}
\left(\nabla \tilde{Y}^{\Re}\right)_{t}^{\ell \cdot}= & \left(\nabla Y^{\Re}\right)_{r_{j+1}^{\ell \cdot}}+\int_{t}^{r_{j+1}}\left(\nabla_{x} f\left(\Theta_{u}^{\Re}\right) \nabla X_{u}+\nabla_{y} f\left(\Theta_{u}^{\Re}\right) \nabla \tilde{Y}_{u}^{\Re}\right. \\
& \left.+\sum_{i=1}^{d} \nabla_{z^{\cdot i}} f^{\ell}\left(\Theta_{u}^{\mathfrak{R}}\right) \nabla Z_{u}^{\mathfrak{R}, i}\right) \mathrm{d} u \\
& -\int_{t}^{r_{j+1}} \sum_{k=1}^{d}\left(\nabla Z_{u}^{\Re, \ell}\right)^{k \cdot} \mathrm{d} W_{u}^{k},
\end{aligned}
$$

where $\left(\nabla Y^{\Re}\right)^{\ell \cdot}$ is defined through the 'pseudo-reflection'

$$
\begin{aligned}
\left(\nabla Y^{\Re}\right)_{t}^{\ell \cdot}:=\left(\nabla \tilde{Y}^{\Re}\right)_{t}^{\ell \cdot}+ & \left(\left(\nabla l^{\ell}\left(X_{t}\right) \nabla X_{t}-\left(\nabla \tilde{Y}^{\Re}\right)_{t}^{\ell \cdot}\right) \mathbf{1}_{\left\{l^{\ell}\left(X_{t}\right) \geq\left(\tilde{Y}_{t}^{\Re}\right)^{\ell}\right\}}\right. \\
& \left.-\left(\left(\nabla \tilde{Y}^{\Re}\right)_{t}^{\ell \cdot}-\nabla h^{\ell}\left(X_{t}\right) \nabla X_{t}\right) \mathbf{1}_{\left\{h^{\ell}\left(X_{t}\right) \leq\left(\tilde{Y}_{t}^{\Re}\right)^{\ell}\right\}}\right) \mathbf{1}_{\{t \in \mathfrak{R}\}} .
\end{aligned}
$$

Observe that the system of coupled BSDEs (3.10) can be rewritten as

$$
\tilde{U}_{t}^{\Re}=U_{r_{j+1}}^{\Re}+\int_{t}^{r_{j+1}} F\left(\nabla X_{u}, \tilde{U}_{u}^{\Re}, V_{u}^{\Re}\right) \mathrm{d} u-\int_{t}^{r_{j+1}} V_{u}^{\Re} \mathrm{d} W_{u}, \quad t \in\left[r_{j}, r_{j+1}\right),
$$

where $F$ is a linear operator with random coefficient and values in $\mathbb{R}^{d^{2}},\left(U^{\Re}, \tilde{U}^{\Re}, V^{\Re}\right)$ takes values in $\mathbb{R}^{d^{2}} \times \mathbb{R}^{d^{2}} \times \mathbb{M}^{d^{2}, d}$, and

$$
\begin{aligned}
& \left(U^{\Re}\right)^{\top}=\left[\left(\nabla Y^{\Re}\right)^{1 \cdot}, \ldots,\left(\nabla Y^{\Re}\right)^{d \cdot}\right], \\
& \left(\tilde{U}^{\Re}\right)^{\top}=\left[\left(\nabla \tilde{Y}^{\Re}\right)^{1 \cdot}, \ldots,\left(\nabla \tilde{Y}^{\Re}\right)^{d \cdot}\right], \\
& \left(V^{\mathfrak{R}}\right)^{\top}=\left[\nabla Z^{\Re, 1}, \ldots, \nabla Z^{\mathfrak{R}, d}\right] .
\end{aligned}
$$

Thus, existence and uniqueness in $f^{2}\left(\mathbb{R}^{d^{2}}\right) \times f^{2}\left(\mathbb{R}^{d^{2}}\right) \times \mathscr{H}^{2}\left(\mathbb{M}^{d^{2}, d}\right)$ follows easily from a simple induction argument.

Remark 3.3. Using (3.2) and (3.8), we observe that $\left(D_{t} Y^{\Re}, D_{t} \tilde{Y}^{\Re},\left(D_{t}\left(Z^{\mathfrak{R}}\right)^{\ell \ell}\right)_{\ell \in\{1, \ldots, d\}}\right)$ and $\left(\nabla Y^{\Re} \nabla X_{t}^{-1} \sigma\left(X_{t}\right), \nabla \tilde{Y}^{\Re} \nabla X_{t}^{-1} \sigma\left(X_{t}\right),\left(\nabla Z^{\mathfrak{R}, \ell} \nabla X_{t}^{-1} \sigma\left(X_{t}\right)\right)_{\ell \in\{1, \ldots, d\}}\right)$ verify the same equation of type (3.11). By uniqueness of the solution, this implies that

$$
\begin{aligned}
D_{t}\left(\tilde{Y}_{s}^{\Re}\right)^{\ell} & =\left(\nabla \tilde{Y}^{\Re}\right)_{s}^{\ell \cdot} \nabla X_{t}^{-1} \sigma\left(X_{t}\right), \\
D_{t}\left(Y_{s}^{\Re}\right)^{\ell} & =\left(\nabla Y^{\Re}\right)_{s}^{\ell \cdot} \nabla X_{t}^{-1} \sigma\left(X_{t}\right), \\
D_{t}\left(Z_{s}^{\Re}\right)^{\cdot \ell} & =\nabla Z_{s}^{\Re, \ell} \nabla X_{t}^{-1} \sigma\left(X_{t}\right),
\end{aligned}
$$

for $\ell \in\{1, \ldots, d\}$ and $t \leq s \leq T$.

Remark 3.4. By using the same arguments as in proof of Proposition 3.2 we easily deduce that, under $(\mathrm{H} f)$ and $(\mathrm{H} r)$,

$$
\left|\nabla \tilde{Y}_{t}^{\Re}\right|^{p}+\left|\nabla Y_{t}^{\Re}\right|^{p} \leq \mathrm{E}_{t}\left[\beta^{p}\right] \text { for } t \leq T \text { and } p \geq 2 .
$$

Recall that $\beta$ denotes an $\mathcal{F}_{T}$-measurable positive random variable whose value may change from line to line, but satisfies $\mathrm{E}\left[\beta^{p}\right] \leq C_{L}^{p}$ for all $p \geq 1$. 


\subsection{Representation in terms of $\left(\nabla X, \nabla \tilde{Y}^{\Re}\right)$}

Combining Corollary 3.2, (3.8), and Remark 3.3, we deduce this last representation for $\left(Z^{\Re}\right)^{\cdot \ell}$.

Corollary 3.3. Let $(H f)$ and $(H r)$ hold. Then, for each $\ell \in\{1, \ldots, d\}$, there is a version of $\left(Z^{\Re}\right)^{\cdot \ell}$ such that

$$
\begin{aligned}
\left(\left(Z_{t}^{\Re}\right)^{\ell \ell}\right)^{\top}=\left(\Lambda_{t}^{\ell}\right)^{-1} \mathrm{E}_{t}\left[\nabla \phi_{\tau_{j}}^{\ell}\left(\Lambda^{\ell} \nabla X\right)_{\tau_{j}}+\int_{t}^{\tau_{j}}\right. & \left(\nabla_{x} f^{\ell}\left(\Theta_{u}^{\Re}\right)\left(\Lambda^{\ell} \nabla X\right)_{u}\right. \\
& \left.\left.+\nabla_{y} f^{\ell}\left(\Theta_{u}^{\Re}\right)\left(\Lambda^{\ell} \nabla \tilde{Y}^{\Re}\right)_{u}\right) \mathrm{~d} u\right] \nabla X_{t}^{-1} \sigma\left(X_{t}\right)
\end{aligned}
$$

for $s \leq t \in\left[r_{j}, r_{j+1}\right)$ and $j \leq \kappa-1$.

\section{Regularity results}

Based on the representations of the previous section and the stability result of Proposition 2.2, we can now provide one of the main results of this paper which concerns the regularity of $\left(Y^{\Re}, \tilde{Y}^{\Re}, Z^{\mathfrak{R}}\right)$. Namely, we study the quantities

$$
\left\|\tilde{Y}^{\mathfrak{R}}-\mathscr{D}^{\pi} \tilde{Y}^{\Re}\right\|_{\mathcal{H}^{2}} \text { and }\left\|Z^{\mathfrak{R}}-P^{\pi} Z^{\mathfrak{R}}\right\|_{\mathscr{H}^{2}},
$$

where $\pi=\left\{0=: t_{0}<t_{1}<\cdots<t_{n}:=T\right\}$ is a partition of the time interval $[0, T]$ with modulus $|\pi|$ and such that $\Re \subset \pi, D^{\pi}$ is the usual piecewise approximation operator defined on $\mathscr{H}^{2}\left(\mathbb{R}^{d}\right)$ by

$$
\mathcal{D}^{\pi} V:=\sum_{i=0}^{n-1} V_{t_{i}} \mathbf{1}_{\left[t_{i}, t_{i+1}\right)}+V_{T} \mathbf{1}_{\{T\}},
$$

and $P^{\pi}$ is defined on $\mathscr{H}^{2}\left(\mathbb{M}^{d}\right)$ by

$$
P^{\pi} V:=\sum_{i=0}^{n-1} \bar{V}_{t_{i}}^{\pi} \mathbf{1}_{\left[t_{i}, t_{i+1}\right)} \quad \text { with } \quad \bar{V}_{t_{i}}^{\pi}:=\frac{1}{t_{i+1}-t_{i}} \mathrm{E}\left[\int_{t_{i}}^{t_{i+1}} V_{s} \mathrm{~d} s \mid \mathcal{F}_{t_{i}}\right] .
$$

Remark 4.1. Here $P^{\pi} V$ is the best $L^{2}(\Omega \times[0, T])$-approximation of $V$ by adapted processes which are constant on each interval $\left[t_{i}, t_{i+1}\right)$.

As shown in [3], [4], [5], and [15], the control of such quantities plays a central role in the study of Euler scheme type approximations of BSDEs and it will be used in the following sections.

\subsection{Regularity of $Y^{\mathfrak{R}}$}

Proposition 4.1. Set $\alpha(\kappa)=\kappa$ under $(H x 1)$ and $(H b 1)$, and $\alpha(\kappa)=1$ under $(H x 1)$, (Hbl), and $(H f)$. Then the following holds:

$$
\sup _{t \in[0, T]} \mathrm{E}\left[\left|\tilde{Y}_{t}^{\Re}-\left(D^{\pi} \tilde{Y}^{\Re}\right)_{t}\right|^{2}\right] \leq C_{L} \alpha(\kappa)|\pi| .
$$

Proof. Noting that, for $j<\kappa$ and $t \in\left[t_{i}, t_{i+1}\right) \subset\left[r_{j}, r_{j+1}\right]$,

$$
\left|\tilde{Y}_{t}^{\Re}-\tilde{Y}_{t_{i}}^{\Re}\right|^{2} \leq 2\left(\int_{t_{i}}^{t_{i+1}}\left|f\left(X_{u}, \tilde{Y}_{u}^{\Re}, Z_{u}^{\Re}\right)\right|^{2} \mathrm{~d} u+\sup _{t \in\left[t_{i}, t_{i+1}\right]}\left|\int_{t}^{t_{i+1}}\left(Z_{u}^{\Re}\right)^{\top} \mathrm{d} W_{u}\right|^{2}\right),
$$


it follows directly from Proposition 2.1, Proposition 4.2, below, and the Burkholder-DavisGundy inequality that

$$
\mathrm{E}\left[\left|\tilde{Y}_{t}^{\Re}-\tilde{Y}_{t_{i}}^{\Re}\right|^{2}\right] \leq C_{L} \alpha(\kappa)|\pi|,
$$

which concludes the proof.

The following immediate corollary provides an estimate of the first term of (4.1).

Corollary 4.1. Set $\alpha(\kappa)=\kappa$ under $(H x 1)$ and $(H b 1)$, and $\alpha(\kappa)=1$ under (Hx 1), (Hb1), and (Hf). Then the following holds:

$$
\left\|\tilde{Y}^{\Re}-\mathscr{D}^{\pi} \tilde{Y}^{\Re}\right\|_{\mathcal{H}^{2}}^{2} \leq C_{L} \alpha(\kappa)|\pi| .
$$

We now state the proposition which was used in the proof of Proposition 4.1. Observe that it provides a 'weak' bound on $Z^{\Re}$.

Proposition 4.2. Set $\alpha(\kappa)=\kappa$ under $(H x 1)$ and $(H b l)$, and $\alpha(\kappa)=1$ under $(H x 1),(H b 1)$, and $(H f)$. There is a version of $Z^{\mathfrak{R}}$ such that the following statements hold.

1. For $s \leq t \leq T$, we have

$$
\mathrm{E}\left[\int_{s}^{t}\left|Z_{u}^{\Re}\right|^{2} \mathrm{~d} u\right] \leq C_{L} \alpha(\kappa)|t-s| .
$$

2. If $(\mathrm{Hr})$ holds then there is a version of $Z^{\mathfrak{R}}$ such that

$$
\mathrm{E}\left[\sup _{t \in[0, T]}\left|Z_{t}^{\Re}\right|^{2}\right] \leq C_{L} \alpha(\kappa) .
$$

Proof. (i) Assume that $(\mathrm{H} r)$ holds. Since $\left(D_{t} \tilde{Y}_{t}^{\Re}\right)_{t \leq T}$ is a version of $\left(Z_{t}^{\Re}\right)_{t \leq T}$, the second claim is a straightforward consequence of Proposition 3.2 and the Burkholder-Davis-Gundy inequality. This implies the first claim under $(\mathrm{H} r)$.

(ii) We now assume that only ( $\mathrm{H} x 1)$ holds for $X$, i.e. $b$ and $\sigma$ are $L$-Lipschitz continuous, and that $(\mathrm{H} b 1)$ holds for $h$ and $l$. Recall that $g$ and $f$ are also $L$-Lipschitz continuous.

Let $\left(f_{n}\right)_{n \geq 0}$ be the sequence of smooth functions defined by

$$
f_{n}(x, y, z)=\int_{\mathbb{R}^{d(d+2)}} \phi_{n}(x-\xi, y-v, z-\zeta) f(\xi, v, \zeta) \mathrm{d} \xi \mathrm{d} v \mathrm{~d} \zeta,
$$

where $\phi_{n}(x, y, z)=n^{d(d+2)} \phi(n(x, y, z))$ and $\phi$ is a compactly supported smooth probability density function on $\mathbb{R}^{d(d+2)}$. Since $f$ is $L$-Lipschitz continuous, we have

$$
\left\|f-f_{n}\right\|_{\infty} \leq \frac{C_{L}}{n} .
$$

Let $g_{n}, \sigma_{n}$, and $b_{n}$ be defined similarly with $f$ replaced by $g, \sigma$, and $b$, respectively, so that

$$
\left\|g-g_{n}\right\|_{\infty}+\left\|b-b_{n}\right\|_{\infty}+\left\|\sigma-\sigma_{n}\right\|_{\infty} \leq \frac{C_{L}}{n} .
$$

Let $X^{n}$ be the diffusion associated to $b_{n}$ and $\sigma_{n}$, and let $\left(Y^{\Re, n}, Z^{\Re, n}\right)$ be the solution of (2.2) associated to $f_{n}, g_{n}$, and $X^{n}$. Since, by step (i) and (H $\left.x 1\right)$,

$$
\mathrm{E}\left[\int_{s}^{t}\left|Z_{u}^{\Re, n}\right|^{2} \mathrm{~d} u\right] \leq C_{L} \alpha(\kappa)|t-s|
$$

for all $s, t \leq T$ and $n \geq 0$, the required result follows from step (i) and Proposition 2.2. 


\subsection{Regularity of $Z^{\mathfrak{R}}$}

The estimate for the second term of (4.1) is a bit more involved. We will adapt the proof of Proposition 5.2 of [3] to our framework.

We first prove a result for the general case. The difficulty, which does not appear in [3], comes from the fact that $D Z^{\mathfrak{R}}$ is in the expression of $Z^{\mathfrak{R}}$ and can be eliminated only when $(\mathrm{H} f$ ) holds. It is overcome using the a priori estimates of the previous section.

Proposition 4.3. Set $\alpha(\kappa)=\kappa$ under $(H x l)$ and $(H b l)$, and $\alpha(\kappa)=1$ under $(H x l),(H b l)$, and $(H f)$. Then the following holds:

$$
\left\|Z^{\Re}-P^{\pi} Z^{\Re}\right\|_{\mathcal{H}^{2}}^{2} \leq C_{L} \alpha(\kappa)\left(\kappa|\pi|+|\pi|^{1 / 2}\right) .
$$

Proof. (i) First observe that a similar approximation argument as the one used in step (ii) of the proof of Proposition 4.2 allows us to reduce our study to the case where ( $\mathrm{H} r$ ) holds. We will therefore assume from now on that $(\mathrm{H} r)$ holds.

Since, by Remark 4.1, $\left\|Z^{\mathfrak{R}}-P^{\pi} Z^{\mathfrak{R}}\right\|_{\mathscr{H}^{2}} \leq\left\|Z^{\mathfrak{R}}-D^{\pi} Z^{\Re}\right\|_{\mathcal{H}^{2}}$, it suffices to show that the last term is bounded by $C_{L} \alpha(\kappa)\left(\kappa|\pi|+|\pi|^{1 / 2}\right)$.

For each $\ell \in\{1, \ldots, d\}$ and $s \leq t \in\left[t_{i}, t_{i+1}\right) \subset\left[r_{j}, r_{j+1}\right]$, we define $V_{s, t}^{\ell, j}$ by

$$
\begin{aligned}
\mathrm{E}_{t}\left[\nabla \phi_{\tau_{j}^{\ell}}^{\ell}\left(D_{s} X\right)_{\tau_{j}^{\ell}}+\int_{s}^{\tau_{j}^{\ell}}(\right. & \nabla_{x} f^{\ell}\left(\Theta_{u}^{\Re}\right) D_{s} X_{u}+\nabla_{y} f^{\ell}\left(\Theta_{u}^{\Re}\right) D_{s} \tilde{Y}_{u}^{\Re} \\
& \left.\left.+\sum_{k=1}^{d} \nabla_{z^{\cdot}} f^{\ell}\left(\Theta_{u}^{\Re}\right) D_{s}\left(Z_{u}^{\mathfrak{R}}\right)^{\cdot k}\right) \mathrm{~d} u\right] .
\end{aligned}
$$

After possibly passing to a suitable version of $Z^{\Re}$, we observe that

$$
\left|\left(Z_{t}^{\Re}\right)^{\cdot \ell}-\left(Z_{t_{i}}^{\Re}\right)^{\cdot \ell}\right| \leq\left|V_{t, t}^{\ell, j}-V_{t_{i}, t}^{\ell, j}\right|+\left|V_{t_{i}, t}^{\ell, j}-V_{t_{i}, t_{i}}^{\ell, j}\right|
$$

(recall Corollary 3.1). Defining $i_{j}$ through $t_{i_{j}}=r_{j}, j \leq \kappa$, we will prove the following controls:

$$
\sum_{j=0}^{\kappa-1} \sum_{k=i_{j}}^{i_{j+1}-1} \int_{t_{k}}^{t_{k+1}} \mathrm{E}\left[\left|V_{t, t}^{\ell, j}-V_{t_{k}, t}^{\ell, j}\right|^{2}\right] \mathrm{d} t \leq C_{L} \alpha(\kappa)|\pi|
$$

and

$$
\sum_{j=0}^{\kappa-1} \sum_{k=i_{j}}^{i_{j+1}-1} \int_{t_{k}}^{t_{k+1}} \mathrm{E}\left[\left|V_{t_{k}, t}^{\ell, j}-V_{t_{k}, t_{k}}^{\ell, j}\right|^{2}\right] \mathrm{d} t \leq C_{L} \alpha(\kappa)\left(\kappa|\pi|+|\pi|^{1 / 2}\right) .
$$

(iia) We first study (4.3). We have, for $t \in\left[t_{i}, t_{i+1}\right) \subset\left[r_{j}, r_{j+1}\right]$,

$$
\begin{aligned}
\left|V_{t, t}^{\ell, j} V_{t_{i}, t}^{\ell, j}\right| \leq C_{L} \mathrm{E}_{t}\left[\int_{t}^{\tau_{j}^{\ell}}\left|D_{t} X_{u}-D_{t_{i}} X_{u}\right|+\left|D_{t} \tilde{Y}_{u}^{\Re}-D_{t_{i}} \tilde{Y}_{u}^{\Re}\right|\right. & \\
& +\sum_{k=1}^{d}\left|D_{t}\left(Z_{u}^{\Re}\right)^{\cdot k}-D_{t_{i}}\left(Z_{u}^{\Re}\right)^{\cdot k}\right| \mathrm{d} u \\
& +\int_{t_{i}}^{t}\left(\left|D_{t_{i}} X_{u}\right|+\left|D_{t_{i}} \tilde{Y}_{u}^{\Re}\right|+\sum_{k=1}^{d}\left|D_{t_{i}}\left(Z_{u}^{\Re}\right)^{\cdot k}\right|\right) \mathrm{d} u \\
& \left.+\left|\nabla \phi_{\tau_{j}^{\ell}}^{\ell}\left(D_{t} X\right)_{\tau_{j}^{\ell}}-\nabla \phi_{\tau_{j}^{\ell}}^{\ell}\left(D_{t_{i}} X\right)_{\tau_{j}^{\ell}}\right|\right]
\end{aligned}
$$


Observing that, by the Cauchy-Schwarz inequality,

$$
\begin{aligned}
& \mathrm{E}\left[\left|\int_{t_{i}}^{t}\left(\left|D_{t_{i}} X_{u}\right|+\left|D_{t_{i}} \tilde{Y}_{u}^{\Re}\right|+\sum_{i=1}^{d}\left|D_{t_{i}}\left(Z_{u}^{\Re}\right)^{\cdot i}\right|\right) \mathrm{d} u\right|^{2}\right] \\
& \quad \leq C_{L}|\pi| \mathrm{E}\left[\int_{t_{i}}^{t}\left(\left|D_{t_{i}} X_{u}\right|^{2}+\left|D_{t_{i}} \tilde{Y}_{u}^{\Re}\right|^{2}+\sum_{k=1}^{d}\left|D_{t_{i}}\left(Z_{u}^{\Re}\right)^{\cdot k}\right|^{2}\right) \mathrm{d} u\right],
\end{aligned}
$$

it follows from Proposition 3.2, Proposition 3.3, (3.3), and (Hr) that

$$
\mathrm{E}\left[\left|V_{t, t}^{\ell, j}-V_{t_{i}, t}^{\ell, j}\right|^{2}\right] \leq C_{L} \alpha(\kappa)|\pi| .
$$

(iib) We now prove (4.4). Using the martingale property of $\left(V_{t_{i}, t}^{\ell, j}\right)_{t \leq T}$ on $\left[t_{i}, t_{i+1}\right]$, we obtain

$$
\begin{aligned}
\mathrm{E}\left[\left|V_{t_{i}, t}^{\ell, j}-V_{t_{i}, t_{i}}^{\ell, j}\right|^{2}\right] & \leq \mathrm{E}\left[\left|V_{t_{i}, t_{i+1}}^{\ell, j}\right|^{2}-\left|V_{t_{i}, t_{i}}^{\ell, j}\right|^{2}\right] \\
& \leq \mathrm{E}\left[\left|V_{t_{i+1}, t_{i+1}}^{\ell, j}\right|^{2}-\left|V_{t_{i}, t_{i}}^{\ell, j}\right|^{2}+\left|V_{t_{i+1}, t_{i+1}}^{\ell, j}-V_{t_{i}, t_{i+1}}^{\ell, j}\right|\left|V_{t_{i+1}, t_{i+1}}^{\ell, j}+V_{t_{i}, t_{i+1}}^{\ell, j}\right|\right],
\end{aligned}
$$

which, by Proposition 3.3, (Hr), the Cauchy-Schwarz inequality, and (4.5), leads to

$$
\mathrm{E}\left[\left|V_{t_{i}, t}^{\ell, j}-V_{t_{i}, t_{i}}^{\ell, j}\right|^{2}\right] \leq \mathrm{E}\left[\left|V_{t_{i+1}, t_{i+1}}^{\ell, j}\right|^{2}-\left|V_{t_{i}, t_{i}}^{\ell, j}\right|^{2}\right]+C_{L} \alpha(\kappa)|\pi|^{1 / 2} .
$$

To conclude the proof of (4.4), it remains to study the first term on the right-hand side of (4.6):

$$
\begin{aligned}
\Sigma^{\ell} & :=\sum_{j=0}^{\kappa-1} \sum_{k=i_{j}}^{i_{j+1}-1} \mathrm{E}\left[\left|V_{t_{k+1}, t_{k+1}}^{\ell, j}\right|^{2}-\left|V_{t_{k}, t_{k}}^{\ell, j}\right|^{2}\right] \\
& =\left(\mathrm{E}\left[\left|V_{T, T}^{\ell, \kappa-1}\right|^{2}-\left|V_{0,0}^{\ell, 0}\right|^{2}\right]+\sum_{j=1}^{\kappa-1} \mathrm{E}\left[\left|V_{r_{j}, r_{j}}^{\ell,-1}\right|^{2}-\left|V_{r_{j}, r_{j}}^{\ell, j}\right|^{2}\right]\right)
\end{aligned}
$$

so that, by Proposition 3.2,

$$
\mathrm{E}\left[\Sigma^{\ell}\right] \leq C_{L} \kappa \alpha(\kappa)
$$

This implies (4.4), completing the proof.

As in the simply reflected case studied in [3], the estimate of Proposition 4.3 can be improved if we impose more regularity on the forward process and the boundaries. The main new difficulty due to our multidimensional setting is that the projection of $\left(Y^{\mathfrak{R}}\right)^{\ell}$ is not well known: it could be equal to the upper or the lower boundary. This is overcome by appealing to the following lemma which is proved at the end of this section.

Lemma 4.1. Recall the definitions of $\nabla \phi^{\ell}$ and $\tau_{j}^{\ell}$ in Proposition 3.2. Under (Hx2), (Hb3), and $(H f)$, the following holds:

$$
\begin{aligned}
& \left|\mathrm{E}_{r_{j}}\left[\nabla \phi_{\tau_{j-1}^{\ell}}^{\ell} \Lambda_{\tau_{j-1}^{\ell}}^{\ell} \nabla X_{\tau_{j-1}^{\ell}}-\nabla \phi_{\tau_{j}^{\ell}}^{\ell} \Lambda_{\tau_{j}^{\ell}}^{\ell} \nabla X_{\tau_{j}^{\ell}}\right]\right| \\
& \quad \leq \mathrm{E}_{r_{j}}\left[\beta \mathbf{1}_{\tau_{j-1}^{\ell}<\tau_{j}^{\ell}=T}\right]+\mathrm{E}_{r_{j}}\left[\beta\left(\tau_{j}^{\ell}-\tau_{j-1}^{\ell}\right)\right]^{1 / 2} \text { for } j<\kappa .
\end{aligned}
$$

This allows us to prove the following proposition. 
Proposition 4.4. If (Hx2), (Hb3), and $(H f)$ hold, then

$$
\left\|Z^{\Re}-P^{\pi} Z^{\Re}\right\|_{\mathscr{H}^{2}}^{2} \leq C_{L} \kappa^{1 / 2}|\pi|
$$

Proof. (i) A similar approximation argument as the one used in step (ii) of the proof of Proposition 4.2 allows us to reduce our study to the case where $(\mathrm{H} r)$ and

- $\sigma$ and $b$ are $C_{b}^{2}$ (smooth version of $(\mathrm{H} x 2)$ ),

- $h$ and $l$ are $C_{b}^{3}$ (smooth version of $(\mathrm{H} b 3)$ ),

hold.

(ii) Under ( $\mathrm{H} f)$, Remark 3.3 implies that

$$
V_{s, t}^{\ell, j}=\eta_{s} \mathrm{E}_{t}\left[A_{s}^{\ell, j}\right], \quad \ell \in\{1, \ldots, d\}, s \leq t \in\left[t_{i}, t_{i+1}\right) \subset\left[r_{j}, r_{j+1}\right],
$$

where

$$
\begin{gathered}
\eta_{s}:=\left(\Lambda_{s}^{\ell} \nabla X_{s}\right)^{-1} \sigma\left(X_{s}\right), \\
A_{s}^{\ell, j}:=\nabla \phi_{\tau_{j}^{\ell}}^{\ell} \Lambda_{\tau_{j}}^{\ell} \nabla X_{\tau_{j}}+\int_{s}^{\tau_{j}^{\ell}}\left(\nabla_{x} f^{\ell}\left(\Theta_{u}^{\Re}\right) \Lambda_{u}^{\ell} \nabla X_{u}+\nabla_{y} f^{\ell}\left(\Theta_{u}^{\Re}\right) \Lambda_{u}^{\ell} \nabla \tilde{Y}_{u}^{\Re}\right) \mathrm{d} u .
\end{gathered}
$$

Recall (4.2) in the proof of Proposition 4.3. We then have to study the quantities

$$
\sum_{j=0}^{\kappa-1} \sum_{k=i_{j}}^{i_{j+1}-1} \int_{t_{k}}^{t_{k+1}} \mathrm{E}\left[\left|V_{t, t}^{\ell, j}-V_{t_{k}, t}^{\ell, j}\right|^{2}\right] \mathrm{d} t \quad \text { and } \sum_{j=0}^{\kappa-1} \sum_{k=i_{j}}^{i_{j+1}-1} \int_{t_{k}}^{t_{k+1}} \mathrm{E}\left[\left|V_{t_{k}, t}^{\ell, j}-V_{t_{k}, t_{k}}^{\ell, j}\right|^{2}\right] \mathrm{d} t
$$

By (4.5) in the proof of Proposition 4.3 applied under $(\mathrm{H} f)$ (i.e. $\alpha(\kappa)=1)$, we first obtain

$$
\sum_{j=0}^{\kappa-1} \sum_{k=i_{j}}^{i_{j+1}-1} \int_{t_{k}}^{t_{k+1}} \mathrm{E}\left[\left|V_{t, t}^{\ell, j}-V_{t_{k}, t}^{\ell, j}\right|^{2}\right] \mathrm{d} t \leq C_{L}|\pi|
$$

To control the second term, we can reproduce line by line the arguments used in the proof of Proposition 5.2 of [3] to obtain

$$
\sum_{j=0}^{\kappa-1} \sum_{k=i_{j}}^{i_{j+1}-1} \int_{t_{k}}^{t_{k+1}} \mathrm{E}\left[\left|V_{t_{k}, t}^{\ell, j}-V_{t_{k}, t_{k}}^{\ell, j}\right|^{2}\right] \mathrm{d} t \leq C_{L}|\pi|\left(1+\Sigma^{\ell}+\left(\tilde{\Sigma}^{\ell}\right)^{1 / 2}\right),
$$

where

$$
\Sigma^{\ell}:=\sum_{j=1}^{\kappa-1} \mathrm{E}\left[\left|V_{r_{j}, r_{j}}^{\ell, j-1}\right|^{2}-\left|V_{r_{j}, r_{j}}^{\ell, j}\right|^{2}\right] \quad \text { and } \quad \tilde{\Sigma}^{\ell}:=\sum_{j=1}^{\kappa-1} \mathrm{E}\left[\left|A_{r_{j}}^{\ell, j-1}\right|^{4}-\left|A_{r_{j}}^{\ell, j}\right|^{4}\right] .
$$

(iii) We now study $\Sigma^{\ell}$ and $\tilde{\Sigma}^{\ell}$. Using (3.7), (3.9) and (3.12), we first obtain

$$
\begin{aligned}
& \left|V_{r_{j}, r_{j}}^{\ell, j-1}\right|^{2}-\left|V_{r_{j}, r_{j}}^{\ell, j}\right|^{2} \\
& \quad \leq \beta\left(\left|\mathrm{E}_{r_{j}}\left[\tau_{j}^{\ell}-\tau_{j-1}^{\ell}\right]\right|+\left|\mathrm{E}_{r_{j}}\left[\nabla \phi_{\tau_{j-1}^{\ell}}^{\ell} \Lambda_{\tau_{j-1}^{\ell}}^{\ell} \nabla X_{\tau_{j-1}^{\ell}}-\nabla \phi_{\tau_{j}^{\ell}}^{\ell} \Lambda_{\tau_{j}^{\ell}}^{\ell} \nabla X_{\tau_{j}^{\ell}}\right]\right|\right),
\end{aligned}
$$


which, by Lemma 4.1, implies that

$$
\Sigma^{\ell} \leq C_{L} \kappa^{1 / 2}
$$

Similar arguments lead to

$$
\tilde{\Sigma}^{\ell} \leq C_{L} \kappa^{1 / 2} .
$$

We conclude the proof by substituting these estimates into (4.7).

Proof of Lemma 4.1. (i) For all $\ell \in\{1 \ldots d\}, j<\kappa$, we introduce

$$
\begin{aligned}
\Delta \phi_{j}^{\ell} & :=\nabla \phi_{\tau_{j-1}^{\ell}}^{\ell} \Lambda_{\tau_{j-1}^{\ell}}^{\ell} \nabla X_{\tau_{j-1}^{\ell}}-\nabla \phi_{\tau_{j}^{\ell}}^{\ell} \Lambda_{\tau_{j}^{\ell}}^{\ell} \nabla X_{\tau_{j}^{\ell}}, \\
\Delta h_{j}^{\ell} & :=\nabla h^{\ell}\left(X_{\tau_{j-1}^{\ell}}\right)_{\tau_{j-1}^{\ell}} \Lambda_{\tau_{j-1}^{\ell}}^{\ell} \nabla X_{\tau_{j-1}^{\ell}}-\nabla h^{\ell}\left(X_{\tau_{j}^{\ell}}\right) \Lambda_{\tau_{j}^{\ell}}^{\ell} \nabla X_{\tau_{j}^{\ell}}, \\
\Delta l_{j}^{\ell}: & =\nabla l^{\ell}\left(X_{\tau_{j-1}^{\ell}}\right)_{\tau_{j-1}^{\ell}} \Lambda_{\tau_{j-1}^{\ell}}^{\ell} \nabla X_{\tau_{j-1}^{\ell}}-\nabla l^{\ell}\left(X_{\tau_{j}^{\ell}}\right) \Lambda_{\tau_{j}^{\ell}}^{\ell} \nabla X_{\tau_{j}^{\ell}} .
\end{aligned}
$$

Since

$$
\nabla \phi_{\tau_{j-1}^{\ell}}^{\ell} \Lambda_{\tau_{j-1}^{\ell}}^{\ell} \nabla X_{\tau_{j-1}^{\ell}}-\nabla \phi_{\tau_{j}^{\ell}}^{\ell} \Lambda_{\tau_{j}^{\ell}}^{\ell} \nabla X_{\tau_{j}^{\ell}}=\Delta \phi_{j}^{\ell}\left(\mathbf{1}_{\left\{\tau_{j-1}^{\ell}<\tau_{j}^{\ell}<T\right\}}+\mathbf{1}_{\left\{\tau_{j-1}^{\ell}<\tau_{j}^{\ell}=T\right\}}\right),
$$

it follows from (3.7) and (3.9) that

$$
\left|\mathrm{E}_{r_{j}}\left[\Delta \phi_{j}^{\ell}\right]\right| \leq \mathrm{E}_{r_{j}}\left[\beta \mathbf{1}_{\left\{\tau_{j-1}^{\ell}<\tau_{j}^{\ell}=T\right\}}\right]+\mathrm{E}_{r_{j}}\left[\left|\Delta \phi_{j}^{\ell}\right| \mathbf{1}_{\left\{\tau_{j-1}^{\ell}<\tau_{j}^{\ell}<T\right\}}\right] .
$$

(ii) We now fix a coordinate $\ell \in\{1, \ldots, d\}$, and set

$$
U_{j}^{\ell}:=\left\{\tau_{j-1}^{\ell}<\tau_{j}^{\ell}<T\right\}, \quad \Delta Y_{j}^{\ell}=\left|\left(\tilde{Y}_{\tau_{j-1}^{\ell}}^{\Re}\right)^{\ell}-\left(Y_{\tau_{j}^{\ell}}^{\Re}\right)^{\ell}\right|, \quad \text { and } \quad \Delta X_{j}=\left|X_{\tau_{j-1}^{\ell}}-X_{\tau_{j}^{\ell}}\right| .
$$

Using the same arguments as in the proof of Proposition 4.1, we obtain

$$
\mathrm{E}_{r_{j}}\left[\left|\Delta Y_{j}^{\ell}\right|^{2}+\left|\Delta X_{j}\right|^{2}\right] \leq \mathrm{E}_{r_{j}}\left[\beta\left(\tau_{j}-\tau_{j-1}\right)\right] .
$$

Since $h^{\ell}$ and $l^{\ell}$ are $L$-Lipschitz continuous and $h^{\ell} \geq l^{\ell}+\varepsilon$, we can find $\eta^{\ell}>0$ and $\varepsilon^{\ell}>0$ such that, for all $x_{1}, x_{2} \in \mathbb{R}^{d}$,

$$
\left|x_{1}-x_{2}\right| \leq \eta^{\ell} \Longrightarrow h\left(x_{1}\right)-l\left(x_{2}\right)>\varepsilon^{\ell} .
$$

Observe that by choosing $L$ large enough we can assume that $1 / \varepsilon \leq L$ so that $1 / \eta^{\ell}+1 / \varepsilon^{\ell} \leq C_{L}$.

We then introduce the three following disjoint sets of $\mathcal{F}_{T}$ :

$$
\begin{aligned}
& A_{j}^{\ell}=\left\{\left|\Delta Y_{j}^{\ell}\right| \leq \varepsilon^{\ell},\left|\Delta X_{j}\right| \leq \eta^{\ell}\right\} \cap U_{j}^{\ell}, \\
& B_{j}^{\ell}=\left\{\left|\Delta Y_{j}^{\ell}\right| \leq \varepsilon^{\ell},\left|\Delta X_{j}\right|>\eta^{\ell}\right\} \cap U_{j}^{\ell}, \\
& C_{j}^{\ell}=\left\{\left|\Delta Y_{j}^{\ell}\right|>\varepsilon^{\ell}\right\} \cap U_{j}^{\ell} .
\end{aligned}
$$

Clearly, $A_{j}^{\ell} \cup B_{j}^{\ell} \cup C_{j}^{\ell}=U_{j}^{\ell}$.

(iiia) On $A_{j}^{\ell} \cap\left\{\left(Y_{\tau_{j}^{\ell}}^{\Re}\right)^{\ell}=h^{\ell}\left(X_{\tau_{j}^{\ell}}\right)\right\}$ we have $\left(Y_{\tau_{j}^{\ell}}^{\Re}\right)^{\ell}-l^{\ell}\left(X_{\tau_{j-1}^{\ell}}\right)>\varepsilon^{\ell}$, by (4.9). But, on $A_{j}^{\ell}$ we also have $\left|\left(Y_{\tau_{j}^{\ell}}^{\Re}\right)^{\ell}-\left(\tilde{Y}_{\tau_{j-1}^{\ell}}^{\Re}\right)^{\ell}\right| \leq \varepsilon^{\ell}$; thus, $\left(Y_{\tau_{j-1}^{\ell}}^{\Re}\right)^{\ell}=h^{\ell}\left(X_{\tau_{j-1}^{\ell}}\right)$. Using the same arguments on $A_{j}^{\ell} \cap\left\{\left(Y_{\tau_{j}^{\ell}}^{\Re}\right)^{\ell}=l^{\ell}\left(X_{\tau_{j}^{\ell}}\right)\right\}$, we obtain $\left(Y_{\tau_{j-1}^{\ell}}^{\Re}\right)^{\ell}=l^{\ell}\left(X_{\tau_{j}^{\ell}}\right)$. Also, since

$$
\left(A_{j}^{\ell} \cap\left\{\left(Y_{\tau_{j}^{\ell}}^{\Re}\right)^{\ell}=h^{\ell}\left(X_{\tau_{j}^{\ell}}\right)\right\}\right) \cup\left(\left(A_{j}^{\ell} \cap\left\{\left(Y_{\tau_{j}^{\ell}}^{\Re}\right)^{\ell}=l^{\ell}\left(X_{\tau_{j}^{\ell}}\right)\right\}\right)=A_{j}^{\ell},\right.
$$


we have

$$
\mathrm{E}_{r_{j}}\left[\left|\Delta \phi_{j}^{\ell}\right| \mathbf{1}_{U_{j}^{\ell}}\right] \leq \mathrm{E}_{r_{j}}\left[\left(\left|\Delta h_{j}^{\ell}\right|+\left|\Delta l_{j}^{\ell}\right|\right) \mathbf{1}_{A_{j}^{\ell}}\right]+\mathrm{E}_{r_{j}}\left[\left|\Delta \phi_{j}^{\ell}\right|\right]\left(\mathbf{1}_{B_{j}^{\ell}}+\mathbf{1}_{C_{j}^{\ell}}\right) .
$$

Using ( $\mathrm{H} b 3)$, we have

$$
\mathrm{E}_{r_{j}}\left[\left(\left|\Delta h_{j}^{\ell}\right|+\left|\Delta l_{j}^{\ell}\right|\right) \mathbf{1}_{A_{j}^{\ell}}\right] \leq \mathrm{E}_{r_{j}}\left[\beta\left(\tau_{j}-\tau_{j-1}\right)\right]^{1 / 2},
$$

and, by Tchebytchev's inequality and (4.8),

$$
\mathrm{E}_{r_{j}}\left[\left|\Delta \phi_{j}^{\ell}\right|\right]\left(\mathbf{1}_{B_{j}^{\ell}}+\mathbf{1}_{C_{j}^{\ell}}\right) \leq \mathrm{E}_{r_{j}}\left[\beta\left(\tau_{j}-\tau_{j-1}\right)\right]^{1 / 2} .
$$

Using (4.10), this leads to

$$
\mathrm{E}_{r_{j}}\left[\left|\Delta \phi_{j}^{\ell}\right| \mathbf{1}_{U_{j}^{\ell}}\right] \leq \mathrm{E}_{r_{j}}\left[\beta\left(\tau_{j}-\tau_{j-1}\right)\right]^{1 / 2},
$$

which concludes the proof.

\section{A discrete-time approximation for discretely reflected BSDEs}

As an application of the regularity results stated in the last section, we now study the convergence of an Euler scheme approximation method for discretely reflected BSDEs. Using an approximation argument, we will then propose an extension of this method to continuously reflected BSDEs in the next section.

\subsection{Discrete-time approximation of the forward process}

As in the previous section, we consider a grid $\pi=\left\{0=: t_{0}<t_{1}<\cdots<t_{n}:=T\right\}$ of the time interval $[0, T]$ with modulus $|\pi|$, such that $\Re \subset \pi$.

As usual, $X$ is approximated by its Euler scheme $X^{\pi}$ defined by

$$
\begin{gathered}
X_{0}^{\pi}=X_{0}, \\
X_{t_{i+1}}^{\pi}=X_{t_{i}}^{\pi}+b\left(X_{t_{i}}^{\pi}\right)\left(t_{i+1}-t_{i}\right)+\sigma\left(X_{t_{i}}^{\pi}\right)\left(W_{t_{i+1}}-W_{t_{i}}\right), \quad i \leq n-1,
\end{gathered}
$$

and, for $t \in\left[t_{i}, t_{i+1}\right), i \leq n-1$,

$$
X_{t}^{\pi}=X_{t_{i}}^{\pi}+b\left(X_{t_{i}}^{\pi}\right)\left(t-t_{i}\right)+\sigma\left(X_{t_{i}}^{\pi}\right)\left(W_{t}-W_{t_{i}}\right) .
$$

Under (Hx1), $b$ and $\sigma$ are $L$-Lipschitz continuous; thus, we have (see, e.g. [13, Chapter 10])

$$
\left\|\sup _{t \leq T}\left|X_{t}-X_{t}^{\pi}\right|\right\|_{L^{p}}+\max _{i<n}\left\|\sup _{t \in\left[t_{i}, t_{i+1}\right]}\left|X_{t}-X_{t_{i}}^{\pi}\right|\right\|_{L^{p}} \leq C_{L}^{p}|\pi|^{1 / 2}, \quad p \geq 1 .
$$

\subsection{An Euler scheme for discretely reflected BSDEs}

We now introduce a discrete-time approximation scheme for the discretely reflected BSDE of the form

$$
\begin{gathered}
\bar{Z}_{t_{i}}^{\pi}=\left(t_{i+1}-t_{i}\right)^{-1} \mathrm{E}_{t_{i}}\left[\left(W_{t_{i+1}}-W_{t_{i}}\right)\left(Y_{t_{i+1}}^{\pi}\right)^{\top}\right], \\
\tilde{Y}_{t_{i}}^{\pi}=\mathrm{E}_{t_{i}}\left[Y_{t_{i+1}}^{\pi}\right]+\left(t_{i+1}-t_{i}\right) f\left(X_{t_{i}}^{\pi}, \tilde{Y}_{t_{i}}^{\pi}, \bar{Z}_{t_{i}}^{\pi}\right), \\
Y_{t_{i}}^{\pi}=\mathcal{R}\left(t_{i}, X_{t_{i}}^{\pi}, \tilde{Y}_{t_{i}}^{\pi}\right), \quad i \leq n-1,
\end{gathered}
$$


with terminal condition

$$
\tilde{Y}_{T}^{\pi}=Y_{T}^{\pi}:=g\left(X_{T}^{\pi}\right) .
$$

This kind of backward scheme has already been considered when no reflection occurs (see, e.g. [5]) and in the simply reflected case (see, e.g. [3], [15], and the references therein).

Combining an induction argument with the Lipschitz continuity of $g, f$, and the projection operator, we can easily check that the above processes are square integrable and that the conditional expectations are well defined at each step of the algorithm.

For later use, we introduce the continuous-time scheme associated to $\left(Y^{\pi}, \bar{Z}^{\pi}\right)$. By the martingale representation theorem, there exists $Z^{\pi} \in \mathcal{H}^{2}\left(\mathbb{M}^{d}\right)$ such that

$$
Y_{t_{i+1}}^{\pi}=\mathrm{E}_{t_{i}}\left[Y_{t_{i+1}}^{\pi}\right]+\int_{t_{i}}^{t_{i+1}}\left(Z_{u}^{\pi}\right)^{\top} \mathrm{d} W_{u}, \quad i \leq n-1 .
$$

We then define $\tilde{Y}^{\pi}$ on $\left[t_{i}, t_{i+1}\right)$ by

$$
\tilde{Y}_{t}^{\pi}=Y_{t_{i+1}}^{\pi}+\left(t_{i+1}-t\right) f\left(X_{t_{i}}^{\pi}, \tilde{Y}_{t_{i}}^{\pi}, \bar{Z}_{t_{i}}^{\pi}\right)-\int_{t}^{t_{i+1}}\left(Z_{u}^{\pi}\right)^{\top} \mathrm{d} W_{u},
$$

and set

$$
Y_{t}^{\pi}:=\mathcal{R}\left(t, X_{t}^{\pi}, \tilde{Y}_{t}^{\pi}\right) \quad \text { for } t \leq T .
$$

We remark that, by the Itô isometry,

$$
\bar{Z}^{\pi}=P^{\pi} Z^{\pi}
$$

where $P^{\pi}$ is defined in (4).

\subsection{Convergence results}

We first provide estimates on the difference between $\left(Y^{\Re}, Z^{\Re}\right)$ and $\left(Y^{\pi}, \bar{Z}^{\pi}\right)$.

Proposition 5.1. Assume that ( $\mathrm{Hxl}$ ) and (Hbl) hold. Then

$$
\begin{aligned}
& \sup _{t \in[0, T]} \mathrm{E}\left[\left|Y_{t}^{\Re}-Y_{t}^{\pi}\right|^{2}\right]+\left\|Z^{\Re}-\bar{Z}^{\pi}\right\|_{\mathcal{H}^{2}}^{2} \\
& \leq C_{L}\left(\left\|\tilde{Y}^{\Re}-\mathscr{D}^{\pi} \tilde{Y}^{\Re}\right\|_{\mathcal{H}^{2}}^{2}+\left\|Z^{\Re}-P^{\pi} Z^{\Re}\right\|_{\mathcal{H}^{2}}^{2} .\right. \\
& \left.\quad+\kappa \mathrm{E}\left[\max _{r \in \mathfrak{R}}\left|X_{r}-X_{r}^{\pi}\right|^{2}\right]+\left\|X-D^{\pi} X^{\pi}\right\|_{\AA^{2}}^{2}\right) .
\end{aligned}
$$

Moreover, if $f^{\ell}$ depends on $(y, z)$ only through $\left(y^{\ell}, z^{\ell}\right)$, we have

$$
\sup _{t \in[0, T]} \mathrm{E}\left[\left|Y_{t}^{\Re}-Y_{t}^{\pi}\right|^{2}\right] \leq C_{L}\left(\left\|\tilde{Y}^{\Re}-D^{\pi} \tilde{Y}^{\Re}\right\|_{\mathcal{H}^{2}}^{2}+\left\|Z^{\Re}-P^{\pi} Z^{\Re}\right\|_{\mathcal{H}^{2}}^{2}+\left\|X-\mathscr{D}^{\pi} X^{\pi}\right\|_{\mathcal{S}^{2}}^{2}\right) .
$$

Before providing the proof of this result, let us observe that combining it with Proposition 4.1, Proposition 4.3, Proposition 4.4, and (5.1), we obtain an upper bound on the approximation error between the Euler scheme (5.2a)-(5.2c) and the discretely reflected BSDE (2.2).

Theorem 5.1. Set $(\alpha(\kappa), \gamma(\kappa))=\left(\kappa^{2}, \kappa\right)$ under $(H x 1)$ and $(H b 1),(\alpha(\kappa), \gamma(\kappa))=(\kappa, 1)$ under (Hx 1), (Hbl), and $(H f)$, and $(\alpha(\kappa), \gamma(\kappa))=(\kappa, 0)$ under $(H x 2),(H b 3)$, and $(H f)$. Then the following holds:

$$
\sup _{t \in[0, T]} \mathrm{E}\left[\left|\tilde{Y}_{t}^{\Re}-\left(\mathscr{D}^{\pi} \tilde{Y}^{\pi}\right)_{t}\right|^{2}\right]+\left\|Z^{\Re}-\bar{Z}^{\pi}\right\|_{\mathscr{H}^{2}}^{2} \leq C_{L}\left(\alpha(\kappa)|\pi|+\gamma(\kappa)|\pi|^{1 / 2}\right) .
$$


Moreover, if (Hx2) and (Hb3) hold, and $f^{\ell}$ depends on $(y, z)$ only through $\left(y^{\ell}, z^{\cdot \ell}\right)$, then we have

$$
\sup _{t \in[0, T]} \mathrm{E}\left[\left|\tilde{Y}_{t}^{\Re}-\left(D^{\pi} \tilde{Y}^{\pi}\right)_{t}\right|^{2}\right] \leq C_{L} \kappa^{1 / 2}|\pi|
$$

Remark 5.1. The estimates above are stated in a fairly general setting. They can be improved in some particular cases.

1. If $X=X^{\pi}$ on $\pi$, i.e. $X$ is 'perfectly simulated', then the term $\mathrm{E}\left[\max _{r \in \mathfrak{R}}\left|X_{r}-X_{r}^{\pi}\right|^{2}\right]=0$ disappears in the estimate of Proposition 5.1. In particular, if $(\mathrm{H} x 2)$ and $(\mathrm{H} b 3)$ hold and $f^{\ell}$ depends on $(y, z)$ only through $\left(y^{\ell}, z^{\ell}\right)$, then we have

$$
\left\|Z^{\Re}-\bar{Z}^{\pi}\right\|_{\mathscr{H}^{2}}^{2} \leq C_{L} \kappa^{1 / 2}|\pi| .
$$

2. If $f$ does not depend on $z$ then

$$
\sup _{t \in[0, T]} \mathrm{E}\left[\left|\tilde{Y}_{t}^{\Re}-\left(\mathscr{D}^{\pi} \tilde{Y}^{\pi}\right)_{t}\right|^{2}\right] \leq C_{L}|\pi| .
$$

This follows from the fact that, in this case, the term $\int_{t_{i-1}}^{t_{i}}\left(\left|Z_{u}^{\Re}-\bar{Z}_{t_{i-1}}^{\Re}\right|^{2}\right) \mathrm{d} u$ in (5.5), below, disappears.

The proof of Proposition 5.1 relies on the following remark.

Remark 5.2. Under $(\mathrm{H} b 1)$, for $t \in \pi$ and each $\ell \in\{1, \ldots, d\}$, there exists $S_{t}^{\ell}$ and $Q_{t}^{\ell}$ in $\mathcal{F}_{t}$ such that $S_{t}^{\ell} \cap Q_{t}^{\ell}=\varnothing$ and

$$
\left|\left(Y_{t}^{\Re}\right)^{\ell}-\left(Y_{t}^{\pi}\right)^{\ell}\right|^{2} \leq\left|\left(\tilde{Y}_{t}^{\Re}\right)^{\ell}-\left(\tilde{Y}_{t}^{\pi}\right)^{\ell}\right|^{2} \mathbf{1}_{S_{t}^{\ell}}+C_{L}\left|X_{t}-X_{t}^{\pi}\right|^{2} \mathbf{1}_{Q_{t}^{\ell}} .
$$

This is shown by arguing as in the proof of Lemma 2.1 .

Moreover, for $t \in[0, T] \backslash \mathfrak{R}$, we have $\left|\left(Y_{t}^{\Re}\right)^{\ell}-\left(Y_{t}^{\pi}\right)^{\ell}\right|=\left|\left(\tilde{Y}_{t}^{\Re}\right)^{\ell}-\left(\tilde{Y}_{t}^{\pi}\right)^{\ell}\right|$ and, for $t \in$ $\pi \backslash \mathfrak{R}$, we can set $S_{t}^{\ell}=\Omega$ and $Q_{t}^{\ell}=\varnothing$.

Proof of Proposition 5.1. We adapt the proof of Theorem 3.1 of [5] to our context.

(ia) We set $\delta Y=Y^{\Re}-Y^{\pi}, \delta \tilde{Y}=\tilde{Y}^{\Re}-\tilde{Y}^{\pi}, \delta Z=Z^{\Re}-\bar{Z}^{\pi}$, and $\delta X=X-X^{\pi}$. Observe that, by (5.3) and Jensen's inequality,

$$
\mathrm{E}\left[\left|\bar{Z}_{t}^{\Re}-\bar{Z}_{t}^{\pi}\right|^{2}\right] \leq\left(t_{i+1}-t_{i}\right)^{-1} \int_{t_{i}}^{t_{i+1}} \mathrm{E}\left[\left|Z_{u}^{\Re}-Z_{u}^{\pi}\right|^{2}\right] \mathrm{d} u,
$$

where $\bar{Z}^{\Re}=P^{\pi} Z^{\mathfrak{R}}$.

Applying Itô's formula to $|\delta \tilde{Y}|^{2}$ on $\left[t_{i}, t_{i+1}\right) \subset\left[r_{j}, r_{j+1}\right)$, using the last inequality and standard arguments (see, e.g. step (i) of Proposition A.1 in Appendix A), we obtain, for all $s \leq t_{i}$,

$$
\begin{aligned}
\mathrm{E}_{S}\left[\left|\delta \tilde{Y}_{t}\right|^{2}+\int_{t}^{t_{i+1}}\left|\delta Z_{u}\right|^{2} \mathrm{~d} u\right] \leq \mathrm{E}_{S}[ & \left|\delta Y_{t_{i+1}}\right|^{2}+\alpha \int_{t}^{t_{i+1}}\left|\delta \tilde{Y}_{u}\right|^{2} \mathrm{~d} u+C_{L} B_{i+1} \\
& \left.+\frac{C_{L}}{\alpha}\left(\left|t_{i+1}-t_{i}\right|\left|\delta \tilde{Y}_{t_{i}}\right|^{2}+\int_{t_{i}}^{t_{i+1}}\left|\delta Z_{u}\right|^{2} \mathrm{~d} u\right)\right],
\end{aligned}
$$

where $\alpha>1$ is to be chosen later on and, for $i \in\{1, \ldots, n\}$,

$$
B_{i}:=\int_{t_{i-1}}^{t_{i}}\left(\left|X_{u}-X_{t_{i-1}}^{\pi}\right|^{2}+\left|\tilde{Y}_{u}^{\Re}-\tilde{Y}_{t_{i-1}}^{\Re}\right|^{2}+\left|Z_{u}^{\mathfrak{R}}-\bar{Z}_{t_{i-1}}^{\Re}\right|^{2}\right) \mathrm{d} u .
$$


By Gronwall's lemma, we deduce that, for all $t \in\left[t_{i}, t_{i+1}\right)$,

$$
\begin{aligned}
\mathrm{E}_{s}\left[\left|\delta \tilde{Y}_{t}\right|^{2}\right] \leq \exp \left\{\alpha C_{L}\left|t_{i+1}-t_{i}\right|\right\} \mathrm{E}_{S}[ & \left|\delta Y_{t_{i+1}}\right|^{2}+C_{L} B_{i+1} \\
& \left.+\frac{C_{L}}{\alpha}\left(\left|t_{i+1}-t_{i}\right|\left|\delta \tilde{Y}_{t_{i}}\right|^{2}+\int_{t_{i}}^{t_{i+1}}\left|\delta Z_{u}\right|^{2} \mathrm{~d} u\right)\right] .
\end{aligned}
$$

Combining the last equation with (5.4), choosing $\alpha$ such that $C_{L} / \alpha \leq \frac{1}{4}$, and then working with $|\pi|$ small enough such that $\alpha|\pi| \exp \left\{C_{L} \alpha|\pi|\right\} \leq 2 \alpha|\pi| \leq 1$, we compute

$$
\mathrm{E}_{S}\left[\left|\delta \tilde{Y}_{t_{i}}\right|^{2}+\frac{1}{2} \int_{t_{i}}^{t_{i+1}}\left|\delta Z_{u}\right|^{2} \mathrm{~d} u\right] \leq \exp \left\{C_{L}\left|t_{i+1}-t_{i}\right|\right\} \mathrm{E}_{s}\left[\left|\delta Y_{t_{i+1}}\right|^{2}+C_{L} B_{i+1}\right]
$$

(ib) For $j \leq \kappa$, we define $i_{j}$ through $t_{i_{j}}=r_{j}$. Since $\left|\delta Y_{t}\right|=\left|\delta \tilde{Y}_{t}\right|$ for all $t \in \pi \backslash \mathfrak{R}$, we deduce from (5.7) and an induction argument that, for $i \in\left[i_{j}, i_{j+1}\right)$,

$$
\mathrm{E}\left[\left|\delta \tilde{Y}_{t_{i}}\right|^{2}\right] \leq \exp \left\{C_{L}\left|r_{j+1}-t_{i}\right|\right\} \mathrm{E}\left[\left|\delta Y_{r_{j+1}}\right|^{2}+C_{L} \sum_{k=i_{j}+1}^{i_{j+1}} B_{k}\right] .
$$

Summing up over $i$ in (5.7), we also obtain

$\mathrm{E}\left[\int_{r_{j}}^{r_{j+1}}\left|\delta Z_{u}\right|^{2} \mathrm{~d} u\right] \leq C_{L} \mathrm{E}\left[\left|\delta X_{r_{j+1}}\right|^{2}+\left|\delta \tilde{Y}_{r_{j+1}}\right|^{2}-\left|\delta \tilde{Y}_{r_{j}}\right|^{2}+|\pi| \sum_{k=i_{j}+1}^{i_{j+1}}\left|\delta \tilde{Y}_{t_{k}}\right|^{2}+\sum_{k=i_{j}+1}^{i_{j+1}} B_{k}\right]$.

Summing up over $j$, this leads to

$$
\mathrm{E}\left[\int_{0}^{T}\left|\delta Z_{u}\right|^{2} \mathrm{~d} u\right] \leq C_{L} \sup _{t \in \pi} \mathrm{E}\left[\left|\delta \tilde{Y}_{t}\right|^{2}+\sum_{i=1}^{n} B_{i}\right]+\kappa \max _{r \in \mathfrak{R}} \mathrm{E}\left[\left|\delta X_{r}\right|^{2}\right]
$$

Using Remark 5.2, (5.8), and an induction argument, we then obtain

$$
\mathrm{E}\left[\left|\delta \tilde{Y}_{r_{j}}\right|^{2}+\left|\delta Y_{r_{j}}\right|^{2}\right] \leq C_{L} \mathrm{E}\left[\left|\delta X_{T}\right|^{2}+\kappa \max _{r \in \mathfrak{R}}\left|\delta X_{r}\right|^{2}+\sum_{q=0}^{\kappa-1} \sum_{k=i_{q}+1}^{i_{q+1}} B_{k}\right], \quad j<\kappa,
$$

which combined with (5.8) leads to

$$
\sup _{i \leq n} \mathrm{E}\left[\left|\delta \tilde{Y}_{t_{i}}\right|^{2}+\left|\delta Y_{t_{i}}\right|^{2}\right] \leq C_{L} \mathrm{E}\left[\kappa \max _{r \in \Re}\left|\delta X_{r}\right|^{2}+\sum_{i=1}^{n} B_{i}\right] .
$$

The proof is then concluded by substituting (5.10) into (5.9), and then combining (5.6) with (5.9) and (5.10).

(ii) We now turn to the case where $f^{\ell}$ depends on $(y, z)$ only through $\left(y^{\ell}, z^{\ell}\right)$.

In this case, (5.6) and (5.7) read as follows:

$$
\begin{aligned}
\mathrm{E}_{s}\left[\left|\left(\delta \tilde{Y}_{t}\right)^{\ell}\right|^{2}\right] \leq & \exp \left\{\alpha C_{L}\left|t_{i+1}-t_{i}\right|\right\} \\
\times \mathrm{E}_{S}\left[\left|\left(\delta Y_{t_{i+1}}\right)^{\ell}\right|^{2}+C_{L} B_{i}\right. & \left.\quad \frac{C_{L}}{\alpha}\left(\left|t_{i+1}-t_{i}\right|\left|\left(\delta \tilde{Y}_{t_{i}}\right)^{\ell}\right|^{2}+\int_{t_{i}}^{t_{i+1}}\left|\left(\delta Z_{u}\right)^{\ell}\right|^{2} \mathrm{~d} u\right)\right]
\end{aligned}
$$


and

$$
\mathrm{E}_{S}\left[\left|\left(\delta \tilde{Y}_{t_{i}}\right)^{\ell}\right|^{2}+\frac{1}{2} \int_{t_{i}}^{t_{i+1}}\left|\left(\delta Z_{u}\right)^{\ell}\right|^{2} \mathrm{~d} u\right] \leq \exp \left\{C_{L}\left|t_{i+1}-t_{i}\right|\right\} \mathrm{E}_{S}\left[\left|\left(\delta Y_{t_{i+1}}\right)^{\ell}\right|^{2}+C_{L} B_{i}\right]
$$

for $t \in\left[t_{i}, t_{i+1}\right), s \leq t_{i}$, and $i<n$.

For each $\ell \in\{1, \ldots, d\}$ and $i<n-1$, we then introduce the sequences of sets $U^{\ell}$ and $\tilde{U}^{\ell}$ defined by

$$
\begin{array}{ccc}
U_{i}^{\ell}:=\Omega & \text { and } \quad U_{i+k}^{\ell}:=U_{i+k-1}^{\ell} \cap S_{t_{i+k}}^{\ell}, \\
\tilde{U}_{i}^{\ell}:=\varnothing \quad \text { and } \quad \tilde{U}_{i+k}^{\ell}:=U_{i+k-1}^{\ell} \cap Q_{t_{i+k}}^{\ell},
\end{array}
$$

for $k \in[1, n-i-1]$.

Recall the definition of $S^{\ell}$ and $Q^{\ell}$ in Remark 5.2. Since $S_{t}^{\ell} \cap Q_{t}^{\ell}=\varnothing$ for each $t$ of $\pi$, we have $U_{i+k}^{\ell} \cap \tilde{U}_{i+k}^{\ell}=\varnothing$ and $\tilde{U}_{i+k}^{\ell} \cap \tilde{U}_{i+j}^{\ell}=\varnothing$ for all $k \in[1, n-i-1]$ and $j \in[k+1, n-i-1]$. Moreover, $U_{i+k}^{\ell}, \tilde{U}_{i+k}^{\ell} \in \tilde{\mathcal{F}}_{t_{i+k}}$.

Using (5.12), Remark 5.2, and an induction argument, we deduce that, for $k \in[1, n-i-1]$,

$$
\mathrm{E}_{t_{i}}\left[\left|\left(\delta \tilde{Y}_{t_{i}}\right)^{\ell}\right|^{2}\right] \leq C_{L} \mathrm{E}_{t_{i}}\left[\left|\left(\delta \tilde{Y}_{t_{i+k+1}}\right)^{\ell}\right|^{2}+\sum_{j=1}^{k}\left(\left|\delta X_{t_{i+j}}\right|^{2} \mathbf{1}_{\tilde{U}_{i+j}^{\ell}}+B_{i+j}\right)\right] .
$$

In particular, for $k=n-i-1$, this leads to

$$
\mathrm{E}_{t_{i}}\left[\left|\left(\delta \tilde{Y}_{t_{i}}\right)^{\ell}\right|^{2}\right] \leq C_{L} \mathrm{E}_{t_{i}}\left[\max _{r \in \Re}\left|\delta X_{r}\right|^{2}+\sum_{j=i+1}^{n} B_{j}\right]
$$

since $\sum_{i=1}^{n-j-1} \mathbf{1}_{\tilde{U}_{i+j}^{\ell}} \leq 1$ and $\left|\delta Y_{T}\right| \leq C_{L}\left|\delta X_{T}\right|$.

Combining the last inequality with (5.11) and (5.12), and using Remark 5.2 again, we obtain

$$
\sup _{t \in[0, T]} \mathrm{E}\left[\left|\left(\delta \tilde{Y}_{t}\right)^{\ell}\right|^{2}+\left|\left(\delta Y_{t}\right)^{\ell}\right|^{2}\right] \leq C_{L} \mathrm{E}\left[\max _{r \in \mathfrak{R}}\left|\delta X_{r}\right|^{2}+\sum_{i=1}^{n} B_{i}\right] .
$$

The proof is then concluded by summing up over $\ell$.

\section{Extensions to continuously reflected BSDEs}

We now apply the results of the last section to continuously reflected BSDEs.

We first obtain a regularity result for the solution of such an equation in the spirit of [15]. We then show that the Euler scheme (5.2a)-(5.2c) can be used to approximate continuously reflected BSDEs, provided that $\Re$ and $\pi$ are conveniently chosen.

In this section we assume the existence and uniqueness of a strong solution to the continuously reflected BSDE defined by

$$
\begin{gathered}
Y_{t}^{\ell}=g^{\ell}\left(X_{T}\right)+\int_{t}^{T} f^{\ell}\left(X_{u}, Y_{u}, Z_{u}\right) \mathrm{d} u-\int_{t}^{T} Z_{u}^{\ell} \mathrm{d} W_{u}+\int_{t}^{T} \mathrm{~d} K_{u}^{\ell+}-\int_{t}^{T} \mathrm{~d} K_{u}^{\ell-}, \\
l^{\ell}\left(X_{t}\right) \leq Y_{t}^{\ell} \leq h^{\ell}\left(X_{t}\right) \quad \text { for all } t \in[0, T], \text { a.s. } \\
\int_{0}^{T}\left(Y_{s}^{\ell}-l^{\ell}\left(X_{s}\right)\right) \mathrm{d} K_{s}^{\ell+}=\int_{0}^{T}\left(Y_{s}^{\ell}-h^{\ell}\left(X_{s}\right)\right) \mathrm{d} K_{s}^{\ell-}=0
\end{gathered}
$$

for each $\ell \in\{1, \ldots, d\}$, where $K^{\ell+}, K^{\ell-} \in 8^{2}(\mathbb{R})$ are continuous and increasing, and $K_{0}^{\ell+}=$ $K_{0}^{\ell-}=0$. 
Remark 6.1. 1 . When $d=1, l$ and $h$ are $C_{b}^{1}$ with $L$-Lipschitz continuous derivatives, and $h \geq l+\varepsilon$ for some $\varepsilon>0$, existence and uniqueness to the above equations are well known; see, e.g. [7]. Obviously this immediately extends to the case where $d>1$ whenever $f^{\ell}$ depends on $(y, z)$ only through $\left(y^{\ell}, z^{\ell}\right)$.

2. When $d \geq 2$, and $h$ and $l$ are constant, existence and uniqueness follow from [10].

The proposition below will allow us to extend the results of the last section to continuously reflected BSDEs. Roughly speaking, it means that $\left(Y^{\mathfrak{R}}, Z^{\mathfrak{R}}\right)$ is a good approximation for $(Y, Z)$.

Proposition 6.1. Set $q=\frac{1}{2}$ under $(H x 1)$ and $(H b 1)$, and $q=1$ under $(H x 1)$, (Hb2), and (Hf). Then we have

$$
\sup _{t \in[0, T]} \mathrm{E}\left[\left|Y_{t}-Y_{t}^{\mathfrak{R}}\right|^{2}\right]+\sup _{t \in[0, T]} \mathrm{E}\left[\left|Y_{t}-\tilde{Y}_{t}^{\Re}\right|^{2}\right]+\left\|Z-Z^{\Re}\right\|_{\mathcal{H}^{2}}^{2} \leq C_{L}|\Re|^{q},
$$

where $|\Re|$ is the modulus of $\Re$.

Proof. First, observe that we can consider each coordinate separately. We can then essentially follow the same arguments as in the proof of Proposition 1.4.1 of [6]. In particular, we have to control both

$$
\int_{t}^{r_{j+1}}\left(l^{\ell}\left(X_{s}\right)-\left(\tilde{Y}_{s}^{\Re}\right)^{\ell}\right) \mathrm{d} K_{s}^{\ell+} \quad \text { and } \quad \int_{t}^{r_{j+1}}\left(\left(\tilde{Y}_{s}^{\Re}\right)^{\ell}-h^{\ell}\left(X_{s}\right)\right) \mathrm{d} K_{s}^{\ell-}, \quad \ell \in\{1, \ldots, d\} .
$$

For all $s \leq T$, we have

$$
\begin{gathered}
l^{\ell}\left(X_{s}\right)-\left(\tilde{Y}_{s}^{\Re}\right)^{\ell} \leq \mathrm{E}_{s}\left[l^{\ell}\left(X_{s}\right)-l^{\ell}\left(X_{r_{j+1}}\right)+\int_{s}^{r_{j+1}}\left|f^{\ell}\left(X_{u}, \tilde{Y}_{u}^{\Re}, Z_{u}^{\Re}\right)\right| \mathrm{d} u\right], \\
\left(\tilde{Y}_{s}^{\Re}\right)^{\ell}-h^{\ell}\left(X_{s}\right) \leq \mathrm{E}_{s}\left[h^{\ell}\left(X_{r_{j+1}}\right)-h^{\ell}\left(X_{s}\right)+\int_{s}^{r_{j+1}}\left|f^{\ell}\left(X_{u}, \tilde{Y}_{u}^{\Re}, Z_{u}^{\Re}\right)\right| \mathrm{d} u\right] .
\end{gathered}
$$

Under $(\mathrm{H} x 1),(\mathrm{H} b 2)$, and $(\mathrm{H} f)$, the control on $h$ and $l$ given by the assumption and the Lipschitz continuity of $\sigma, b$, and $f$, imply that

$$
\begin{aligned}
l^{\ell}\left(X_{s}\right)-\left(\tilde{Y}_{s}^{\Re}\right)^{\ell} \leq & C_{L} \mathrm{E}_{s}\left[\int_{s}^{r_{j+1}}\left(1+\left|\rho_{1}^{\ell}\left(X_{s}\right)^{\top} b\left(X_{u}\right)\right|+\left|\rho_{3}^{\ell}\left(X_{s}\right)\right|\left(1+\left|X_{u}\right|^{2}\right)\right) \mathrm{d} u\right] \\
& +C_{L} \mathrm{E}_{s}\left[\int_{s}^{r_{j+1}}\left(\left|X_{u}\right|+\left|\tilde{Y}_{u}^{\Re}\right|+\left|Z_{u}^{\Re}\right|\right) \mathrm{d} u\right] .
\end{aligned}
$$

It then follows, from the Cauchy-Schwarz inequality and Propositions 2.1 and 4.2, that

$$
l^{\ell}\left(X_{s}\right)-\left(\tilde{Y}_{s}^{\Re}\right)^{\ell} \leq|\Re| \beta .
$$

Similar arguments applied to (6.2) lead to

$$
\left(\tilde{Y}_{s}^{\Re}\right)^{\ell}-h^{\ell}\left(X_{s}\right) \leq|\Re| \beta .
$$

Under ( $\mathrm{H} x 1)$ and $(\mathrm{H} b 1)$, we use the Lipschitz continuity of $l$ to obtain

$$
l^{\ell}\left(X_{s}\right)-\left(\tilde{Y}_{s}^{\Re}\right)^{\ell} \leq C_{L} \mathrm{E}_{s}\left[L\left|X_{s}-X_{r_{j+1}}\right|+\int_{s}^{r_{j+1}}\left(\left|X_{u}\right|+\left|\tilde{Y}_{u}^{\Re}\right|+\left|Z_{u}^{\Re}\right|\right) \mathrm{d} u\right] .
$$


It then follows, from Proposition 2.1, Proposition 4.2, and the Cauchy-Schwarz inequality, that

$$
l^{\ell}\left(X_{s}\right)-\left(\tilde{Y}_{s}^{\Re}\right)^{\ell} \leq\left(|\Re|^{1 / 2}+\kappa^{1 / 2}|\mathfrak{R}|\right) \beta \leq|\mathfrak{R}|^{1 / 2} \beta .
$$

Similarly, we have

$$
\left(\tilde{Y}_{s}^{\Re}\right)^{\ell}-h^{\ell}\left(X_{s}\right) \leq|\Re|^{1 / 2} \beta .
$$

In both cases, the proof is then concluded by arguing exactly as in [3].

Combining this proposition with Proposition 4.1 and Proposition 4.3, we deduce the following regularity property for $(Y, Z)$.

Corollary 6.1. Set $q=\frac{1}{3}$ under (Hxl) and (Hbl), $q=\frac{1}{2}$ under (Hx l), (Hbl), and (Hf), and $q=1$ under (Hx 1), (Hb2), and (Hf). Then the following holds:

$$
\sup _{t \in[0, T]} \mathrm{E}\left[\left|Y_{t}-\left(\mathcal{D}^{\pi} Y\right)_{t}\right|^{2}\right] \leq C_{L}|\pi|^{q} \text { and }\left\|Z-P^{\pi} Z\right\|_{\mathcal{H}^{2}}^{2} \leq C_{L}|\pi|^{q / 2} .
$$

Moreover, if $q=\frac{1}{3} \operatorname{under}(H x 1),(H b 1)$, and $(H f)$, and $q=\frac{2}{3} \operatorname{under}(H x 2),(H b 3)$, and $(H f)$, then we have

$$
\left\|Z-P^{\pi} Z\right\|_{\mathscr{H}^{2}}^{2} \leq C_{L}|\pi|^{q} .
$$

Proof. (i) We first study the regularity of $Y$. Since,

$$
\sup _{t \in[0, T]} \mathrm{E}\left[\left|Y_{t}-\left(\mathcal{D}^{\pi} Y\right)_{t}\right|^{2}\right] \leq C_{L}\left(\sup _{t \in[0, T]} \mathrm{E}\left[\left|\tilde{Y}_{t}^{\Re}-\left(\mathcal{D}^{\pi} \tilde{Y}^{\Re}\right)_{t}\right|^{2}\right]+\sup _{t \in[0, T]} \mathrm{E}\left[\left|Y_{t}-\tilde{Y}_{t}^{\Re}\right|^{2}\right]\right),
$$

the bound on $\sup _{t \in[0, T]} \mathrm{E}\left[\left|Y_{t}-\left(D^{\pi} Y\right)_{t}\right|^{2}\right]$ is obtained by applying Proposition 4.1 and Proposition 6.1 , with $\Re$ and $\pi$ chosen such that

$$
|\mathfrak{R}| \leq \frac{C_{L}}{\kappa} \quad \text { and } \quad|\Re|=O\left(|\pi|^{\alpha}\right)
$$

for $\alpha=\frac{2}{3}$ under ( $\left.\mathrm{H} x 1\right)$ and $(\mathrm{H} b 1), \alpha=1$ under $(\mathrm{H} x 1),(\mathrm{H} b 1)$, and $(\mathrm{H} f)$, and $\alpha=\frac{1}{2}$ under ( $\mathrm{H} x 1),(\mathrm{H} b 2)$, and $(\mathrm{H} f)$.

(ii) We now turn to $Z$. By Jensens's inequality we have

$$
\left\|Z-P^{\pi} Z\right\|_{\mathcal{H}^{2}}^{2} \leq C_{L}\left(\left\|Z^{\mathfrak{R}}-P^{\pi} Z^{\Re}\right\|_{\mathscr{H}^{2}}^{2}+\left\|Z-Z^{\Re}\right\|_{\mathscr{H}^{2}}^{2}\right) .
$$

Thus, choosing $\mathfrak{R}$ and $\pi$ as in (6.3) with $\alpha=\frac{1}{3}$ under $(\mathrm{H} x 1)$ and $(\mathrm{H} b 1), \alpha=\frac{2}{3}$ under $(\mathrm{H} x 1)$, $(\mathrm{H} b 1)$, and $(\mathrm{H} f), \alpha=\frac{1}{2}$ under $(\mathrm{H} x 1),(\mathrm{H} b 2)$, and $(\mathrm{H} f), \alpha=\frac{2}{3}$ under $(\mathrm{H} x 2),(\mathrm{H} b 3)$, and $(\mathrm{H} f)$, we obtain the required bound by combining Proposition 4.3 with Proposition 6.1.

We now state the main result of this section, which provides an upper bound for the convergence rate of the Euler scheme (5.2a)-(5.2c) to the continuously reflected BSDE (6.1a)(6.1c).

Theorem 6.1. Set $q=\frac{1}{6}$ under (Hxl) and (Hbl), $q=\frac{1}{3}$ under (Hxl), (Hbl), and $(H f)$, $q=\frac{1}{2}$ under (Hx2), (Hb3), and $(H f)$. Then we have

$$
\sup _{t \in[0, T]} \mathrm{E}\left[\left|Y_{t}-\left(\mathscr{D}^{\pi} Y^{\pi}\right)_{t}\right|^{2}\right]+\left\|Z-\bar{Z}^{\pi}\right\|_{\mathcal{H}^{2}}^{2} \leq C_{L}|\pi|^{q}
$$

Moreover, if (Hx2), (Hb3), and (Hf) hold and $X^{\pi}=X$ on $\pi$, then

$$
\sup _{t \in[0, T]} \mathrm{E}\left[\left|Y_{t}-\left(D^{\pi} Y^{\pi}\right)_{t}\right|^{2}\right]+\left\|Z-\bar{Z}^{\pi}\right\|_{\mathcal{H}^{2}}^{2} \leq C_{L}|\pi|^{2 / 3} .
$$


Proof. This is a direct consequence of Proposition 6.1 and Theorem 5.1 applied with $\mathfrak{R}$ and $\pi$ defined as in (6.3), with $\alpha=\frac{1}{3}$ under $(\mathrm{H} x 1)$ and $(\mathrm{H} b 1), \alpha=\frac{2}{3}$ under $(\mathrm{H} x 1),(\mathrm{H} b 1)$, and $(\mathrm{H} f), \alpha=\frac{1}{2}$ under $(\mathrm{H} x 2),(\mathrm{H} b 3)$, and $(\mathrm{H} f)$, and $\alpha=\frac{2}{3}$ under $(\mathrm{H} x 2),(\mathrm{H} b 3)$, and $(\mathrm{H} f)$, and when $X^{\pi}=X$ on $\pi$.

The results of the last theorem can be compared to those of Theorem 4.1 of [3], which gives an upper bound for the rate of convergence in the case of unidimensional simply reflected BSDEs.

First, observe that $(\mathrm{H} b 1)$ is weaker than the assumptions of Theorem 4.1 of [3] and the price to pay for these fairly mild regularity assumptions is the poor rate of convergence.

Second, under $(\mathrm{H} x 2),(\mathrm{H} b 3)$, and $(\mathrm{H} f)$, we are not able to retrieve the result of [3]. This can be explained by the structure of $f$ in our multidimensional setting. In particular, its dependence with respect to all components of $y$ prevents us from getting rid of the term $\kappa \mathrm{E}\left[\max _{r \in \mathfrak{R}} \mid X_{r}-\right.$ $\left.\left.X_{r}^{\pi}\right|^{2}\right]$ in the first claim of Proposition 5.1.

Let us conclude this paper with the following result which deals with the special case when the system of BSDEs is decoupled.

Theorem 6.2. Assume that $f^{\ell}$ depends on $(y, z)$ only through $\left(y^{\ell}, z^{\ell}\right)$, and set $q=\frac{1}{2}$ under (Hx l) and (Hb2), and $q=\frac{2}{3}$ under (Hx2) and (Hb3). Then we have

$$
\sup _{t \in[0, T]} \mathrm{E}\left[\left|Y_{t}-\left(\mathcal{D}^{\pi} Y^{\pi}\right)_{t}\right|^{2}\right] \leq C_{L}|\pi|^{q} .
$$

Proof. This is an immediate consequence of Proposition 6.1 and the second claim of Theorem 5.1 applied with $\Re$ and $\pi$ defined as in (6.3), with $\alpha=\frac{1}{2}$ under $(\mathrm{H} x 1)$ and (H $\left.b 2\right)$, and $\alpha=\frac{2}{3}$ under $(\mathrm{H} \times 2)$ and $(\mathrm{H} b 3)$.

Note that, when $d=1$, the last restriction on $f$ holds trivially. In this case, $Y$ can be interpreted as the price of a game option (see, e.g. [14]). This provides an interesting financial application of our result.

Also, observe that, in Theorem 6.2, we obtain better bounds on the convergence rate. But, we are not able to retrieve the bounds of [3], due to the presence of two reflecting boundaries; see Lemma 4.1.

\section{Appendix A. A priori estimates}

In this section we provide a priori estimates for reflected BSDEs in an abstract framework. We consider processes $\left(Y^{\Re}, \tilde{Y}^{\Re}, Z^{\Re}\right) \in 8^{2}\left(\mathbb{R}^{d}\right) \times \delta^{2}\left(\mathbb{R}^{d}\right) \times \mathscr{H}^{2}\left(\mathbb{M}^{d}\right)$ such that

$$
\tilde{Y}_{t}^{\Re}=Y_{r_{j+1}}^{\Re}+\int_{t}^{r_{j+1}} \hat{f}(u) \mathrm{d} u-\int_{t}^{r_{j+1}}\left(Z_{u}^{\mathfrak{R}}\right)^{\top} \mathrm{d} W_{u}, \quad t \in\left[r_{j}, r_{j+1}\right), j<\kappa,
$$

where $\hat{f}$ is some adapted process satisfying

$$
|\hat{f}| \leq C_{L}\left(|\eta|+\left|\tilde{Y}^{\Re}\right|+\left|Z^{\Re}\right|\right) \quad \text { for some } \eta \in \mathscr{H}^{2}(\mathbb{R}) .
$$

We also assume that

$$
\left|\tilde{Y}_{t}^{\Re}\right|=\left|Y_{t}^{\Re}\right| \quad \text { for all } t \notin \Re \backslash T,
$$

and we work under the following assumption. 
(A0) For all $\ell \in\{1, \ldots, d\}$ and $r \in \mathfrak{R}$,

$$
\left|\left(Y_{r}^{\Re}\right)^{\ell}\right| \leq\left|\left(\tilde{Y}_{r}^{\Re}\right)^{\ell}\right| \mathbf{1}_{S_{r}^{\ell}}+\left|\xi_{r}^{\ell}\right| \mathbf{1}_{Q_{r}^{\ell}}
$$

with $\xi \in s^{2}(\mathbb{R}), S_{r}^{\ell}, Q_{r}^{\ell} \in \mathcal{F}_{r}, S_{r}^{\ell} \cap Q_{r}^{\ell}=\varnothing$, and $S_{T}^{\ell}=\varnothing$.

Obviously, this implies that

$$
\left|Y_{r}^{\Re}\right|^{2} \leq\left|\tilde{Y}_{r}^{\Re}\right|^{2}+\left|\xi_{r}\right|^{2}, \quad r \in \Re, \quad \text { and } \quad\left|Y_{T}^{\Re}\right|^{2} \leq\left|\xi_{T}\right|^{2} .
$$

We will also make use of the following assumption, which is a particular case of (A.2).

(A $f$ ) For each $\ell \in\{1, \ldots, d\}$ and all $u \in[0, T]$, we have

$$
\left|\hat{f}^{\ell}(u)\right| \leq C_{L}\left(\left|\eta_{u}\right|+\left|\tilde{Y}_{u}^{\Re}\right|+\left|\left(Z_{u}^{\Re}\right)^{\cdot \ell}\right|\right) .
$$

In this framework, we can state the following proposition.

Proposition A.1. Under (AO), for all $s \leq T$, the following holds:

$$
\sup _{t \in[s, T]} \mathrm{E}_{S}\left[\left|\tilde{Y}_{t}^{\Re}\right|^{2}+\int_{t}^{T}\left|Z_{u}^{\Re}\right|^{2} \mathrm{~d} u\right] \leq C_{L} \mathrm{E}_{s}\left[\kappa \max _{r \in \Re}\left|\xi_{r}\right|^{2}+\int_{0}^{T}\left|\eta_{u}\right|^{2} \mathrm{~d} u\right] .
$$

When, moreover, (Af) holds, we have

$$
\sup _{t \in[s, T]} \mathrm{E}_{s}\left[\left|\tilde{Y}_{t}^{\Re}\right|^{2}\right] \leq C_{L} \mathrm{E}_{s}\left[\max _{r \in \Re}\left|\xi_{r}\right|^{2}+\int_{0}^{T}\left|\eta_{u}\right|^{2} \mathrm{~d} u\right], \quad s \leq T,
$$

and, for all $s \leq t, t \in\left[r_{j}, r_{j+1}\right)$, and $j<\kappa$,

$$
\mathrm{E}_{S}\left[\int_{t}^{\tau_{j}^{\ell}}\left|\left(Z_{u}^{\Re}\right)^{\ell}\right|^{2} \mathrm{~d} u\right] \leq C_{L} \mathrm{E}_{S}\left[\max _{r \in \mathfrak{R}}\left|\xi_{r}\right|^{2}+\int_{0}^{T}\left|\eta_{u}\right|^{2} \mathrm{~d} u\right],
$$

where

$$
\tau_{j}^{\ell}=\inf \left\{r \in \Re \mid r \geq r_{j+1}, \mathbf{1}_{Q_{r}^{\ell}} \neq 0\right\} \wedge T, \quad j \leq \kappa-1, \ell \leq d .
$$

Proof. (i) Since $\tilde{Y}^{\Re} \in s^{2}\left(\mathbb{R}^{d}\right)$, applying Itô's formula to $\left|\tilde{Y}^{\Re}\right|^{2}$ on $\left[r_{j}, r_{j+1}\right)$ implies that

$$
\mathrm{E}_{s}\left[\left|\tilde{Y}_{t}^{\Re}\right|^{2}+\int_{t}^{r_{j+1}}\left|Z_{u}^{\Re}\right|^{2} \mathrm{~d} u\right]=\mathrm{E}_{s}\left[\left|Y_{r_{j+1}}^{\Re}\right|^{2}+2 \int_{t}^{r_{j+1}}\left\langle\tilde{Y}_{u}^{\Re}, \hat{f}(u)\right\rangle \mathrm{d} u\right]
$$

for all $s \leq t \in\left[r_{j}, r_{j+1}\right), j<\kappa$.

Fix $\alpha>1$ to be chosen later on. Combining the Cauchy-Schwarz inequality and (A.2) with the inequality $a b \leq \alpha a^{2}+b^{2} / \alpha, \alpha>0$, we compute, for all $s \leq t$,

$$
\begin{aligned}
\mathrm{E}_{s}\left[\left|\tilde{Y}_{t}^{\Re}\right|^{2}+\int_{t}^{r_{j+1}}\left|Z_{u}^{\mathfrak{R}}\right|^{2} \mathrm{~d} u\right] \leq \mathrm{E}_{S}[ & \left|Y_{r_{j+1}}^{\Re}\right|^{2}+(\alpha+1) C_{L} \int_{t}^{r_{j+1}}\left|\tilde{Y}_{u}^{\Re}\right|^{2} \mathrm{~d} u \\
& \left.+\frac{C_{L}}{\alpha} \int_{t}^{r_{j+1}}\left(\left|Z_{u}^{\mathfrak{R}}\right|^{2}+\left|\eta_{u}\right|^{2}\right) \mathrm{d} u\right] .
\end{aligned}
$$


Taking $\alpha$ large enough such that $C_{L} / \alpha \leq \frac{1}{2}$, we obtain

$$
\mathrm{E}_{s}\left[\left|\tilde{Y}_{t}^{\Re}\right|^{2}+\frac{1}{2} \int_{t}^{r_{j+1}}\left|Z_{u}^{\Re}\right|^{2} \mathrm{~d} u\right] \leq \mathrm{E}_{s}\left[\left|Y_{r_{j+1}}^{\Re}\right|^{2}+C_{L} \int_{t}^{r_{j+1}}\left(\left|\tilde{Y}_{u}^{\Re}\right|^{2}+\left|\eta_{u}\right|^{2}\right) \mathrm{d} u\right] .
$$

Using Gronwall's lemma in the last inequality, we then obtain

$$
\begin{aligned}
& \mathrm{E}_{S}\left[\left|\tilde{Y}_{t}^{\Re}\right|^{2}+\frac{1}{2} \int_{t}^{r_{j+1}}\left|Z_{u}^{\Re}\right|^{2} \mathrm{~d} u\right] \\
& \quad \leq \exp \left\{C_{L}\left|r_{j+1}-t\right|\right\} \mathrm{E}_{S}\left[\left|Y_{r_{j+1}}^{\Re}\right|^{2}+C_{L} \int_{t}^{r_{j+1}}\left|\eta_{u}\right|^{2} \mathrm{~d} u\right] \quad \text { for all } s \leq t \in\left[r_{j}, r_{j+1}\right) .
\end{aligned}
$$

(ii) It follows easily, from (A.5), (A.3), (A0), and an induction argument, that

$$
\sup _{t \in[s, T]} \mathrm{E}_{s}\left[\left|\tilde{Y}_{t}^{\Re}\right|^{2}+\left|Y_{t}^{\Re}\right|^{2}\right] \leq C_{L} \mathrm{E}_{S}\left[\left|\tilde{Y}_{T}^{\Re}\right|^{2}+\kappa \max _{r \in \Re}\left|\xi_{r}\right|^{2}+\int_{s}^{T}\left|\eta_{u}\right|^{2} \mathrm{~d} u\right] \quad \text { for all } s \leq T \text {. }
$$

Moreover, (A.5) applied to $t=r_{j}$ and $s \leq r_{j}$ reads, recall (A.4),

$$
\begin{aligned}
& \mathrm{E}_{S}\left[\left|\tilde{Y}_{r_{j}}^{\Re}\right|^{2}+\frac{1}{2} \int_{r_{j}}^{r_{j+1}}\left|Z_{u}^{\Re}\right|^{2} \mathrm{~d} u\right] \\
& \quad \leq\left(1+C_{L}|\Re|\right) \mathrm{E}_{S}\left[\left|\tilde{Y}_{r_{j+1}}^{\Re}\right|^{2}+\left|\xi_{r_{j+1}}\right|^{2}+C_{L} \int_{r_{j}}^{r_{j+1}}\left|\eta_{u}\right|^{2} \mathrm{~d} u\right] \text { for } j<\kappa .
\end{aligned}
$$

Summing up in this inequality and using (A.6), we obtain

$$
\mathrm{E}_{S}\left[\int_{t}^{T}\left|Z_{u}^{\Re}\right|^{2} \mathrm{~d} u\right] \leq C_{L} \mathrm{E}_{S}\left[\left|\tilde{Y}_{T}^{\Re}\right|^{2}+\kappa \max _{r \in \mathfrak{R}}\left|\xi_{r}\right|^{2}+\int_{t}^{T}\left|\eta_{u}\right|^{2} \mathrm{~d} u\right], \quad s \leq t \leq T,
$$

which concludes the proof of the first claim.

(iii) We now turn to the case where (A $f$ ) holds. Recalling (A.1) and applying Itô's formula to $\left|\left(\tilde{Y}^{\Re}\right)^{\ell}\right|^{2}$ on $\left[r_{j}, r_{j+1}\right)$, we obtain

$$
\begin{aligned}
\left|\left(\tilde{Y}_{t}^{\Re}\right)^{\ell}\right|^{2}+\int_{t}^{r_{j+1}}\left|\left(Z_{u}^{\Re}\right)^{\ell \ell}\right|^{2} \mathrm{~d} u= & \left|\left(Y_{r_{j+1}}^{\Re}\right)^{\ell}\right|^{2}-2 \int_{t}^{r_{j+1}}\left(\tilde{Y}_{u}^{\Re}\right)^{\ell}\left(\left(Z_{u}^{\Re}\right)^{\ell \ell}\right)^{\top} \mathrm{d} W_{u} \\
& +2 \int_{t}^{r_{j+1}}\left(\tilde{Y}_{u}^{\Re}\right)^{\ell} \hat{f}^{\ell}(u) \mathrm{d} u
\end{aligned}
$$

for all $s \leq t \in\left[r_{j}, r_{j+1}\right), j<\kappa$.

Recall the definition of $\tau_{q}^{\ell}$. On $\left\{\tau_{q}^{\ell}=r_{q+1}\right\}$ we obviously have

$$
\begin{aligned}
\left|\left(\tilde{Y}_{t}^{\Re}\right)^{\ell}\right|^{2}+\int_{t}^{\tau_{j}^{\ell}}\left|\left(Z_{u}^{\Re}\right)^{\ell \ell}\right|^{2} \mathrm{~d} u \leq & \left|\xi_{\tau_{j}^{\ell}}\right|^{2}-2 \int_{t}^{\tau_{j}^{\ell}}\left(\tilde{Y}_{u}^{\Re}\right)^{\ell}\left(\left(Z_{u}^{\Re}\right)^{\cdot \ell}\right)^{\top} \mathrm{d} W_{u} \\
& +2 \int_{t}^{\tau_{j}^{\ell}}\left(\tilde{Y}_{u}^{\Re}\right)^{\ell} \hat{f}^{\ell}(u) \mathrm{d} u .
\end{aligned}
$$

On $\left\{\tau_{q}^{\ell}>r_{q+1}\right\}$ we denote by $\theta_{q}^{\ell}$ the random index such that $r_{\theta_{q}^{\ell}}=\tau_{q}^{\ell}$. Summing up from $q$ to $\theta_{q}^{\ell}$ in (A.7) applied to $t=r_{q+1}$, we retrieve the last inequality. 
Arguing as in step (i), recall (A $f)$, we then obtain

$$
\begin{aligned}
& \mathrm{E}_{S}\left[\left|\left(\tilde{Y}_{t}^{\Re}\right)^{\ell}\right|^{2}+\frac{1}{2} \int_{t}^{\tau_{q}^{\ell}}\left|\left(Z_{u}^{\Re}\right)^{\cdot \ell}\right|^{2} \mathrm{~d} u\right] \\
& \quad \leq C_{L} \mathrm{E}_{S}\left[\left|\xi_{\tau_{q}^{\ell}}\right|^{2}+\int_{t}^{\tau_{q}^{\ell}}\left(\left|\tilde{Y}_{u}^{\Re}\right|^{2}+\left|\eta_{u}\right|^{2}\right) \mathrm{d} u\right] \text { for all } s \leq t \in\left[r_{q}, r_{q+1}\right), q<\kappa .
\end{aligned}
$$

Summing up over $\ell$ in the last inequality, we obtain

$$
\mathrm{E}_{s}\left[\left|\tilde{Y}_{t}^{\Re}\right|^{2}\right] \leq C_{L} \mathrm{E}_{s}\left[\max _{r \in \mathfrak{R}}\left|\xi_{r}\right|^{2}+\int_{t}^{T}\left(\left|\tilde{Y}_{u}^{\Re}\right|^{2}+\left|\eta_{u}\right|^{2}\right) \mathrm{d} u\right], \quad s \leq t \in\left[r_{j}, T\right] .
$$

Using Gronwall's lemma, we then have

$$
\sup _{t \in[s, T]} \mathrm{E}_{s}\left[\left|\tilde{Y}_{t}^{\Re}\right|^{2}\right] \leq C_{L} \mathrm{E}_{s}\left[\max _{r \in \Re}\left|\xi_{r}\right|^{2}+\int_{0}^{T}\left|\eta_{u}\right|^{2} \mathrm{~d} u\right], \quad s \leq T .
$$

Combining this inequality with (A.8), we also obtain

$$
\mathrm{E}_{s}\left[\int_{t}^{\tau_{j}^{\ell}}\left|\left(Z_{u}^{\mathfrak{R}}\right)^{\ell \ell}\right|^{2} \mathrm{~d} u\right] \leq C_{L} \mathrm{E}_{s}\left[\max _{r \in \mathfrak{R}}\left|\xi_{r}\right|^{2}+\int_{t}^{T}\left|\eta_{u}\right|^{2} \mathrm{~d} u\right],
$$

which concludes the proof.

Corollary A.1. Fix $p \geq 2$, and assume that $\xi \in \AA^{p}(\mathbb{R})$ and $\eta \in L^{p}(\Omega \times[0, T])$. Then when (Af) holds, we have, for all $t \leq T$,

$$
\left|\tilde{Y}_{t}^{\Re}\right|^{p} \leq C_{L}^{p} \mathrm{E}_{t}\left[\max _{r \in \Re}\left|\xi_{r}\right|^{p}+\int_{0}^{T}\left|\eta_{u}\right|^{p} \mathrm{~d} u\right] .
$$

Proof. This follows directly from Jensen's inequality applied to (A.9) with $t=s$.

\section{References}

[1] Bahlali, K., Hamadène, S. and Mezerdi, B. (2005). Backward stochastic differential equations with two reflecting barriers and continuous with quadratic growth coefficient. Stoch. Process. Appl. 115, 1107-1129.

[2] Bally, V. And Pagès, G. (2003). Error analysis of the quantization algorithm for obstacle problems. Stoch. Process. Appl. 106, 1-40.

[3] Bouchard, B. and Chassagneux, J.-F. (2008). Discrete-time approximation for continuously and discretely reflected BSDEs. Stoch. Process. Appl. 118, 2269-2293.

[4] Bouchard, B. AND EliE, R. (2008). Discrete-time approximation of decoupled forward-backward SDE with jumps. Stoch. Process. Appl. 118, 53-75.

[5] Bouchard, B. AND Touzi, N. (2004). Discrete-time approximation and Monte-Carlo simulation of backward stochastic differential equations. Stoch. Process. Appl. 111, 175-206.

[6] Chassagneux, J.-F. (2008). Processus réfléchis en finance et probabilité numérique. Doctoral Thesis, Université Paris Diderot - Paris 7.

[7] Cvitanić, J. and Karatzas, I. (1996). Backward stochastic differential equations with reflection and Dynkin games. Ann. Prob. 24, 2024-2056.

[8] El Karoui, N., Peng, S. and Quenez, M. C. (1997). Backward stochastic differential equations in finance. Math. Finance 7, 1-71.

[9] El Karoui, N. et al. (1997). Reflected solutions of backward SDE's, and related obstacle problems for PDE's. Ann. Prob. 25, 702-737.

[10] Gegout-Petit, A. And Pardoux, E. (1996). Equations différentielles stochastiques rétrogrades réfléchies dans un convexe. Stoch. Stoch. Reports 57, 111-128. 
[11] Lemor, J.-P., Gobet E. AND WARIN, X. (2006). Rate of convergence of an empirical regression method for solving generalized backward stochastic differential equations. Bernoulli 12, 889-916

[12] Kifer, Y. (2000). Game options. Finance Stoch. 4, 443-463.

[13] Kloeden, P. E. And Platen, E. (1992). Numerical Solution of Stochastic Differential Equations (Appl. Math. 23). Springer, Berlin.

[14] Ma, J. AND Cvitanić, J. (2001). Reflected forward-backward SDEs and obstacle problems with boundary conditions. J. Appl. Math. Stoch. Anal. 14, 113-138.

[15] Ma, J. AND Zhang, J. (2005). Representations and regularities for solutions to BSDEs with reflections. Stoch. Process. Appl. 115, 539-569.

[16] Nualart, D. (1995). The Malliavin Calculus and Related Topics. Springer, New York.

[17] Zhang, J. (2001). Some fine properties of backward stochastic differential equations. Doctoral Thesis, Purdue University. 